\title{
Gravity-mediated (or composite) Dark Matter confronts astrophysical data
}

\author{
Hyun Min Lee, ${ }^{a}$ Myeonghun Park ${ }^{b, c}$ and Verónica Sanz ${ }^{d}$ \\ ${ }^{a}$ Department of Physics, Chung-Ang University, \\ Seoul 156-756, Korea \\ ${ }^{b}$ Kavli Institute for the Physics and Mathematics of the Universe (WPI), \\ Todai Institutes for Advanced Study, The University of Tokyo, \\ Japan \\ ${ }^{c}$ Theory Division, Physics Department, CERN, \\ CH-1211 Geneva 23, Switzerland \\ ${ }^{d}$ Department of Physics and Astronomy, University of Sussex, \\ Brighton BN1 9QH, U.K. \\ E-mail: hminlee@cau.ac.kr, myeonghun.park@cern.ch, v.sanz@sussex.ac.uk
}

ABSTRACT: We consider the astrophysical bounds on a new form of dark matter, the so called Gravity-mediated Dark Matter. In this scenario, dark matter communicates with us through a mediator sector composed of gravitational resonances, namely a new scalar (radion) and a massive spin-two resonance (massive graviton). We consider specific models motivated by natural electroweak symmetry breaking or weak-scale dark matter in the context of models in warped extra-dimensions and their composite duals. The main Dark Matter annihilation mechanism is due to the interactions of KK gravitons to gauge bosons that propagate in bulk. We impose the bounds on monochromatic or continuum photons from Fermi-LAT and HESS. We also explore scenarios in which the Fermi gamma-ray line could be a manifestation of Gravity-mediated Dark Matter.

Keywords: Beyond Standard Model, Cosmology of Theories beyond the SM, Field Theories in Higher Dimensions, Large Extra Dimensions

ARXIV EPRINT: 1401.5301 


\section{Contents}

1 Introduction 1

2 Setup 2

2.1 KK graviton mediator 4

2.2 Radion mediator 6

3 Dark matter annihilations with KK graviton mediator $\quad 6$

3.1 Scalar dark matter $\quad 9$

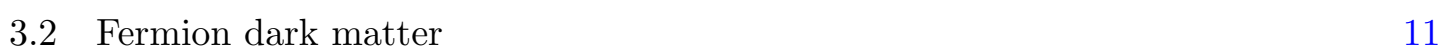

$\begin{array}{lll}3.3 & \text { Vector dark matter } & 13\end{array}$

$\begin{array}{lll}3.4 & \text { Impact of Higgs portal couplings } & 14\end{array}$

$\begin{array}{ll}3.5 & \text { The Fermi-LAT line } \\ \end{array}$

4 Astrophysical constraints $\quad 16$

$\begin{array}{ll}4.1 \text { Monochromatic photons } & 18\end{array}$

$\begin{array}{ll}4.2 \text { Continuum photons } & 18\end{array}$

$\begin{array}{lll}4.3 \text { Gamma-ray boxes } & 19\end{array}$

$\begin{array}{llr}5 & \text { Direct detection and collider bounds } & 19\end{array}$

6 Conclusions 20

\section{Introduction}

Indirect detection experiments with cosmic rays are a testing ground for the signatures of dark matter(DM) annihilation or decay from the gamma-ray and anti-particle production. In particular, in the case of dark matter annihilation, Weakly Interacting Massive Particles(WIMPs) can provide easily the necessary thermal cross section for the relic density of about $\langle\sigma v\rangle \simeq 3 \times 10^{-26} \mathrm{~cm}^{3} / \mathrm{s}$ from the thermal freezeout and can be tested by complementarity between direct and indirect detection experiments. In order for indirect detection to be relevant, dark matter annihilation should not depend on the temperature much, namely, showing the s-wave behavior.

Recently there has been an interesting indication for dark matter from the gammaray line at about $130 \mathrm{GeV}$ coming from the galactic center in Fermi-LAT data [1-5]. The significance of the gamma-ray line has become smaller with reprocessed data than the previous result to $3.3 \sigma$, being reduced to 1.6 at the global level [5]. Nonetheless, it remains to be seen, how the gamma-ray line signal evolves in a near future. The observed Fermi gamma-ray line implies that the annihilation cross section of dark matter into monochromatic photons is $\langle\sigma v\rangle=(1.27-2.27) \times 10^{-27} \mathrm{~cm}^{3} / \mathrm{s}[1]$, depending on whether dark matter 
profile is given by Einasto or NFW. But, the corresponding process is loop-suppressed as compared to the tree-level processes, so it is challenging to build a microscopic model for dark matter interactions [6-31]. Furthermore, the tree-level DM annihilations have been strongly constrained by the gamma-ray searches [32-43] and anti-proton bounds [44-46]. Independent of whether the Fermi gamma-ray line survives more data, the gamma-ray constraints are getting more important for dark matter model building in general.

In this paper, we consider a gravity-mediated dark matter, that has been proposed by the authors [47] to relate dark matter mass to the geometric solution of the hierarchy problem in the 5D warped extra dimension with UV and IR branes [48]. We generalize the previous setup to Model A and B, depending on whether the Higgs doublet is localized on IR or UV branes. In both models, the SM particles propagate in bulk while dark matter of arbitrary spin $(s=0,1 / 2$ and 1$)$ is localized on the IR brane. When the SM fermions are localized toward the UV brane as is the case for fermion mass hierarchy and natural flavor conservation, dark matter annihilates mainly into gauge bosons living in the bulk. In Model B, we show that the Fermi gamma-ray line at about $130 \mathrm{GeV}$ can be obtained from the annihilation of a pair of vector dark matter into a photon pair, which is mediated by the KK graviton without a need of large couplings or resonance. We present the astrophysical bounds from Fermi-LAT and HESS on the models and relate them to the direct detection of dark matter and the discovery potential of the KK graviton at the LHC.

The paper is organized as follows. We begin with the setups for dark matter in the $5 \mathrm{D}$ warped spacetime and compute the annihilation cross sections of scalar, fermion and vector dark matter in the models. Then, we search the parameter space for KK graviton coupling and mass, being consistent with the relic density and impose the astrophysical constraints on the model. In next section, the direct detection and the collider signatures will be discussed. Finally, conclusions are drawn.

\section{Setup}

In this section, we present the general couplings of the KK graviton and the radion to the SM particles, that are determined by the locations of the SM particles in a warped gravitational background with two branes.

There are two setups that are distinguished by the location of the Higgs fields depicted in figure 1:

1. Model A - Hierarchy problem: Higgs fields and dark matter are localized on the IR brane while the SM matter is localized on the UV brane.

2. Model B - WIMP dark matter: dark matter is localized on the IR brane while the $S M$ matter and Higgs fields are localized on the UV brane.

In both models, gauge fields are assumed to propagate in bulk. Dark matter has a strong coupling to the KK graviton and the radion, that are localized on the same brane. In Model A, dark matter can annihilate dominantly into Higgs degrees of freedom. When top quark is localized towards the IR brane, dark matter can annihilate into a top quark 

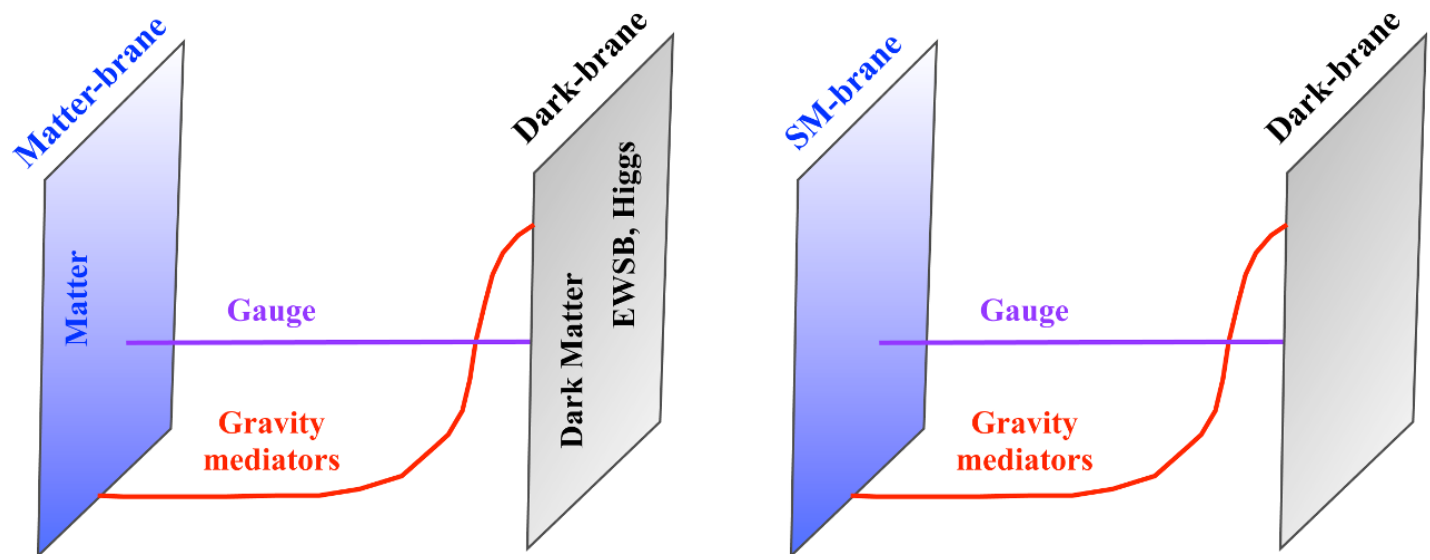

Figure 1. Two setups for dark matter in extra-dimensions. Left (Model A): Matter-brane and Dark-brane are at the opposite sides of the extra dimension while Higgs fields are on the Darkbrane. Right (Model B): the Dark-brane and Standard Model brane are at the opposite sides of the extra-dimension. Gauge fields live in the bulk in both cases and Dark Matter communicates to the SM via the gravity mediators.

pair with sizable branching fraction, if kinematically allowed. On the other hand, in Model $\mathrm{B}$, the annihilation of dark matter into a pair of the SM matter or Higgs fields is suppressed so dark matter can annihilate dominantly into a pair of SM gauge bosons, leading to a large branching fraction of dark matter annihilation into a photon pair.

In the RS background [48], the graviton and the radion are described by the tensor and scalar fluctuations of the warped metric,

$$
d s^{2}=w(z)^{2}\left(e^{-2 r}\left(\eta_{\mu \nu}+G_{\mu \nu}\right)-(1+2 r)^{2} d z^{2}\right),
$$

where $w(z)=1 /(k|z|)$ is the warp factor and $G_{\mu \nu}$ and $r$ are 5D fields propagating in the extra dimension. The fifth dimension is compactified to an interval $z \in\left[z_{0}, z_{1}\right]$, and fourdimensional branes with nonzero tensions are located at the ends of the extra dimension.

The present model in extra-dimensions can be interpreted in terms of a stronglycoupled model in four-dimensions. Some details of this duality can be found in ref. [47].

In a nutshell, the strongly coupled $4 \mathrm{D}$ physics evolution from high to low energies is encoded in the values of the parameters when measured at a position $z$ in the extradimension. The brane at $z_{0}$ represents UV boundary conditions to this evolution, and the brane at $z_{1}$ corresponds to the IR boundary conditions. Propagation from the Matter towards the Dark brane is equivalent to integrate out degrees of freedom. At a position $z_{*}$ the local cutoff is related to the $4 \mathrm{D}$ UV cutoff as [49] $\Lambda\left(z_{*}\right)=\omega\left(z_{*}\right) \Lambda_{U V}$. The IR brane encodes information on the physics leading to confinement, and can be used to engineer the spontaneous breaking of a $4 \mathrm{D}$ global symmetry due to the strong sector.

Kaluza-Klein states are the dual of bound states due to confinement physics. Localization near the UV (IR) brane means a small (large) degree of compositeness of the state. De-localized (flat) gauge fields in the extra-dimension represent global symmetries of the composite sector, weakly gauged by the UV dynamics [50]. These flat fields are a mixture of composite and elementary fields, in analogy with the $\rho-\gamma$ mixing in QCD [51-54]. 
The presence of gravity mediators is a manifestation of a conformal symmetry of the composite sector, which is spontaneously broken by the strong physics. The radion is dual to the goldstone boson from dilatation symmetry in 4D [55-57], the dilaton. The dual interpretation of the massive graviton is not so clear. We interpret the massive KK graviton as a manifestation of a CFT diffeomorphism invariance, broken spontaneously by the Dark Brane, but a more rigorous investigation should be done to understand the dual role of the massive graviton. See ref. [47] for more details.

\subsection{KK graviton mediator}

We introduce the interactions of the SM particles and dark matter to the KK graviton $G_{\mu \nu}$ as

$$
\begin{aligned}
\mathcal{L}_{\mathrm{KK}}= & -\frac{1}{\Lambda} G^{\mu \nu}\left[T_{\mu \nu}^{\mathrm{DM}}+c_{\psi}^{G}\left(\frac{i}{4} \bar{\psi}\left(\gamma_{\mu} D_{\nu}+\gamma_{\nu} D_{\mu}\right) \psi-\frac{i}{4}\left(D_{\mu} \bar{\psi} \gamma_{\nu}+D_{\nu} \bar{\psi} \gamma_{\nu}\right) \psi\right.\right. \\
& \left.-g_{\mu \nu}\left(\bar{\psi} \gamma^{\mu} D_{\mu} \psi-m_{\psi} \bar{\psi} \psi\right)+\frac{i}{2} g_{\mu \nu} \partial^{\rho}\left(\bar{\psi} \gamma_{\rho} \psi\right)\right) \\
& +c_{V}^{G}\left(\frac{1}{4} g_{\mu \nu} F^{\lambda \rho} F_{\lambda \rho}-F_{\mu \lambda} F_{\nu}^{\lambda}\right) \\
& \left.+c_{H}^{G}\left(-g_{\mu \nu} D^{\rho} H^{\dagger} D_{\rho} H+g_{\mu \nu} V(H)+D_{\mu} H^{\dagger} D_{\nu} H+D_{\nu} H^{\dagger} D_{\mu} H\right)\right]
\end{aligned}
$$

with the energy-momentum tensor for dark matter (DM) given by ${ }^{1}$

$$
\begin{aligned}
T_{\mu \nu}^{(\text {Vector DM })} & =\frac{1}{4} g_{\mu \nu} X^{\lambda \rho} X_{\lambda \rho}-X_{\mu \lambda} X_{\nu}^{\lambda}+m_{X}^{2}\left(X_{\mu} X_{\nu}-\frac{1}{2} g_{\mu \nu} X^{\lambda} X_{\lambda}\right) \\
T_{\mu \nu}^{(\text {Fermion DM })} & =\frac{i}{4} \bar{\chi}\left(\gamma_{\mu} \partial_{\nu}+\gamma_{\nu} \partial_{\mu}\right) \chi-\frac{i}{4}\left(\partial_{\mu} \bar{\chi} \gamma_{\nu}+\partial_{\nu} \bar{\chi} \gamma_{\nu}\right) \chi-g_{\mu \nu}\left(i \bar{\chi} \gamma^{\mu} \partial_{\mu} \chi-m_{\chi} \bar{\chi} \chi\right) \\
& +\frac{i}{2} g_{\mu \nu} \partial^{\rho}\left(\bar{\chi} \gamma_{\rho} \chi\right) \\
T_{\mu \nu}^{\text {(Scalar DM })} & =\partial_{\mu} S \partial_{\nu} S-\frac{1}{2} g_{\mu \nu} \partial^{\rho} S \partial_{\rho} S+\frac{1}{2} g_{\mu \nu} m_{S}^{2} S^{2}
\end{aligned}
$$

Here, $c_{X, \chi, S}^{G}, c_{V}^{G}, c_{\psi}^{G}, c_{H}^{G}$ are KK graviton couplings which are determined by the overlap between the wave functions of the KK graviton and fields in extra dimensions, see refs. [59-69] for an example in AdS. $X(\chi, S), V, H$ and $\psi$ denote the Dark Matter particle, gauge bosons, Higgs and SM matter fields, respectively. When the KK graviton mediator connects between dark matter and the SM particles, the DM annihilations are s-wave for scalar and vector dark matters whereas they are p-wave for fermion dark matter. Thus, only scalar and vector dark matters lead to observable gamma-ray signatures at present.

The KK graviton couplings to the electroweak gauge bosons are written schematically: for transverse modes,

$$
G\left(c_{W}^{G} W_{T} W_{T}+c_{B}^{G} B_{T} B_{T}\right)=G\left(c_{\gamma \gamma} A_{T} A_{T}+c_{Z \gamma} Z_{T} A_{T}+c_{Z Z} Z_{T} Z_{T}+c_{W W} W_{T}^{+} W_{T}^{-}\right),
$$

\footnotetext{
${ }^{1}$ Note that at the level of interactions of the spin-two particle with two SM particles, the structure of the coupling is identical whether it is a massive KK-graviton or a resonance from a new 4D strongly coupled sector. This has been shown in ref. [58], and it is a consequence of Lorentz, gauge and CP invariance.
} 
with

$$
\begin{aligned}
c_{\gamma \gamma} & =c_{B}^{G} \cos ^{2} \theta_{W}+c_{W}^{G} \sin ^{2} \theta_{W}, \\
c_{Z \gamma} & =\left(c_{W}^{G}-c_{B}^{G}\right) \sin \left(2 \theta_{W}\right), \\
c_{Z Z} & =c_{W}^{G} \cos ^{2} \theta_{W}+c_{B}^{G} \sin ^{2} \theta_{W}, \\
c_{W W} & =2 c_{W}^{G},
\end{aligned}
$$

and for longitudinal modes,

$$
c_{H}^{G} G\left(m_{W}^{2} W_{L}^{+} W_{L}^{-}+m_{Z}^{2} Z_{L} Z_{L}\right) .
$$

Thus, for the universal gravity couplings to electroweak gauge bosons, $c_{W}^{G}=c_{B}^{G}=c_{V}^{G}$, the $Z \gamma$ coupling vanishes, so there is no DM annihilation into $Z \gamma$ with graviton mediator. ${ }^{2}$ For comparison, non-gravitational interactions of singlet pseudo-scalar or extra gauge boson mediator lead to the DM annihilation into $Z_{\gamma}$ [29-31], which is an extra source for monochromatic photons. ${ }^{3}$

In warped extra-dimensions, there is a hierarchy of couplings of the graviton to Dark matter, Bulk, Matter and Higgs fields, respectively. Indeed, in our setup, one obtains

Dark matter: $c_{X}^{G} \simeq \mathcal{O}(1)$,

$$
\begin{aligned}
\text { Bulk fields: } \quad c_{V}^{G} \simeq \frac{1}{\int_{\text {Datter }}^{\text {Matt }} w(z) d z}, \\
\text { Matter fields: } \quad c_{\psi}^{G}=\left(\frac{z_{\text {Matter }}}{z_{\text {Dark }}}\right)^{\alpha}, \\
\text { Higgs fields: } \quad c_{H}^{G} \simeq \mathcal{O}(1) /\left(\frac{z_{\text {Matter }}}{z_{\text {Dark }}}\right)^{\alpha} \text { Model A / B }
\end{aligned}
$$

where $\alpha>1$. In AdS models, the value of $c_{V}^{G}$ is

$$
c_{V}^{G}=2 \frac{1-J_{0}\left(x_{G}\right)}{\log \left(\frac{M_{P l}}{\mathrm{TeV}}\right) x_{G}^{2}\left|J_{2}\left(x_{G}\right)\right|}
$$

where $x_{G}=3.83$ is the first zero of the Bessel function $J_{1}$, given in the absence of localized kinetic terms. Here we see explicitly the suppression by $\left(\int w(z) d z\right)^{-1}=1 / \log \left(M_{P} / \mathrm{TeV}\right) \simeq$ $\mathcal{O}(0.03)$.

For simplicity, we have shown the effect of exchanging one KK-mode. In the next sections we will also present results including the effect of the whole KK-tower. This can be done for any metric of the form of eq. (2.1) as shown in [77]. See ref. [47] for more details.

\footnotetext{
${ }^{2}$ This result could change if localized kinetic terms [70,71] are introduced, but their effect is naturally suppressed.

${ }^{3}$ In the case of box-shaped gamma-ray spectrum [72-74], $Z \gamma$ in the final states comes from the decay of intermediate states that dark matter annihilates into. In this case, $Z \gamma$ channel depends on whether the intermediate state decays into $Z \gamma$ or not.
} 


\subsection{Radion mediator}

The radion of extra dimensions, $r$, couples to the trace of the energy-momentum tensor [47, 78] as follows,

$$
\begin{aligned}
\mathcal{L}_{\text {dilaton }}= & \frac{1}{\sqrt{6} \Lambda} r T_{\mu}^{\mu} \\
= & \frac{1}{\sqrt{6} \Lambda} r\left[T^{\mathrm{DM}}+c_{\psi}^{r}\left(-\frac{7}{2} \bar{\psi} i \gamma^{\mu} D_{\mu} \psi-\frac{1}{2} D_{\mu} \bar{\psi} i \gamma^{\mu} \psi+4 m_{\psi} \bar{\psi} \psi+2 \partial^{\mu}\left(\bar{\psi} i \gamma_{\mu} \psi\right)\right)\right. \\
& \left.+c_{H}^{r}\left(2 D^{\mu} H^{\dagger} D_{\mu} H-4 V(H)\right)-\sum_{a} \frac{\beta_{a}\left(g_{a}\right)}{2 g_{a}} F_{\mu \nu}^{a} F^{a \mu \nu}\right]
\end{aligned}
$$

with

$$
\begin{aligned}
T^{(\text {Vector DM })} & =-c_{X}^{r} m_{X}^{2} X_{\mu} X^{\mu}, \\
T^{(\text {Fermion DM })} & =c_{\chi}^{r}\left(-3 i \bar{\chi} \gamma^{\mu} \partial_{\mu} \chi+4 m_{\chi} \bar{\chi} \chi-\frac{5}{2} \partial^{\mu}\left(\bar{\chi} i \gamma_{\mu} \chi\right)\right), \\
T^{(\text {Scalar DM })} & =c_{S}^{r}\left(-\partial^{\mu} S \partial_{\mu} S+2 m_{S}^{2} S^{2}\right) .
\end{aligned}
$$

Here, the radion couplings are denoted by $c_{X, \chi, X}^{r}, c_{\psi}^{r}, c_{H}^{r}$, which are determined by an overlap between the wave functions of the radion and the fields in the extra dimension, similarly as for the KK graviton couplings.

We note that including the linear radion couplings, non-derivative radion interactions to massive scalar and vector particles are fixed by dilatation symmetry $[47,78]$ to

$$
\begin{aligned}
\mathcal{L}_{\text {non-deriv }}= & -\left(\frac{r}{\sqrt{6} \Lambda}-\frac{r^{2}}{6 \Lambda^{2}}\right)\left(c_{H}^{r} m_{A}^{2} A_{\mu} A^{\mu}+c_{X}^{r} m_{X}^{2} X_{\mu} X^{\mu}\right) \\
& +2\left(\frac{r}{\sqrt{6} \Lambda}-\frac{r^{2}}{3 \Lambda^{2}}\right)\left(c_{H}^{r} m_{h}^{2} h^{2}+c_{S}^{r} m_{S}^{2} S^{2}\right) .
\end{aligned}
$$

When the radion connects between dark matter and the SM particles, the DM annihilations are s-wave for scalar and vector dark matters whereas they are p-wave for fermion dark matter. In the radion case, we note that the interactions to gauge bosons induced by trace anomalies are loop-suppressed, but there exist tree-level couplings to massive gauge bosons after electroweak symmetry breaking. Thus, the radion decay into a photon pair is loop-suppressed, so is the DM annihilation into a photon pair with radion mediator. Therefore, we could not explain the Fermi gamma-ray line with the radion mediator only.

\section{Dark matter annihilations with KK graviton mediator}

In this section, assuming that the KK graviton mediator contributes dominantly to the annihilation processes for dark matter of any spin, we show how the coupling and mass of the KK graviton are constrained by the relic density and the indirect detection experiments. We also discuss the effect of Higgs portal couplings on the relic density and consider the possibility of explaining the Fermi gamma-ray line in our models. 
First we briefly discuss the model dependence of the dark matter annihilation cross section into a photon pair and the branching fractions of other annihilation channels. In Model A, where the SM fermions are localized on the UV brane while gauge bosons propagate in bulk and Higgs boson is localized on the IR brane, we get $c_{H}^{G}=\mathcal{O}(1) \gg c_{V}^{G}=$ $c_{g}^{G}=c_{W}^{G}=c_{B}^{G} \simeq 0.03 \gg c_{\psi}^{G}$. In this case, the KK graviton has strong couplings to Higgs boson and longitudinal components of $W, Z$ gauge bosons on the IR brane, while it has suppressed couplings to the transverse components of gauge bosons and the SM fermions. Then, the branching fraction of the KK graviton decay rate into a photon pair is highly suppressed,

$$
\frac{\Gamma_{G}(\gamma \gamma)}{\Gamma_{G}(\text { total })} \simeq \frac{\left(c_{B}^{G}\right)^{2}}{8\left(c_{g}^{G}\right)^{2}+3\left(c_{W}^{G}\right)^{2}+\left(c_{B}^{G}\right)^{2}+\left(c_{H}^{G}\right)^{2}} \simeq 10^{-3} .
$$

Thus, assuming the s-channel dominance for DM annihilations, the branching fraction of the DM annihilation into a photon pair to the total cross section is negligibly small in Model A.

On the other hand, in Model B, all the SM gauge bosons live in bulk, so $c_{V}^{G}=c_{g}^{G}=$ $c_{W}^{G}=c_{B}^{G} \simeq 0.03$, whereas the SM fermions and the Higgs doublet are localized at the UV brane so their couplings to the KK graviton are suppressed as $c_{\psi}^{G}, c_{H}^{G} \ll c_{V}^{G}$. Then, the KK graviton decays dominantly into the transverse modes of the SM gauge bosons, so there is a definite prediction for the branching fractions for the KK graviton decay. In particular, for universal gauge couplings with $c_{g}^{G}=c_{W}^{G}=c_{B}^{G}$, the branching fraction of the $\gamma \gamma$ decay mode is about

$$
\frac{\Gamma_{G}(\gamma \gamma)}{\Gamma_{G}(\text { total })} \simeq \frac{\left(c_{B}^{G}\right)^{2}}{8\left(c_{g}^{G}\right)^{2}+3\left(c_{W}^{G}\right)^{2}+\left(c_{B}^{G}\right)^{2}}=\frac{1}{12} \simeq 0.083 .
$$

Consequently, ignoring $W / Z$ masses and taking only the s-channels with KK graviton mediator for $m_{\mathrm{DM}}<m_{G}$, the ratio of the DM annihilation cross section into $\gamma \gamma$ to the total cross section is roughly the same as the branching fraction of the corresponding KK graviton decay rate and it is about $0.083,{ }^{4}$ which could be compatible with the Fermi gamma-ray line $[1,5]$. For $m_{\mathrm{DM}}>m_{G}$, dark matter can also annihilate into a pair of KK gravitons, so the branching fractions of annihilation cross sections are changed. In the case of a sizable KK graviton coupling to gluons, the KK graviton can be produced copiously by gluon fusion at the LHC, while the KK graviton to diphoton rate is greater than the Higgs to diphoton rate. Thus, we can constrain the KK graviton coupling to gluons by the Higgs-like boson search at the LHC.

In figure 2, the branching fractions of the KK graviton decay rates are shown as a function of KK graviton mass for Model A and B, respectively. In the upper panel of figure 2, we have omitted the decay mode of the KK graviton into a pair of dark matter, assuming that it is kinematically disallowed. In Model A and $\mathrm{B}$, below the $W W$ threshold, the KK graviton decays into a gluon pair with about $90 \%$ or a photon pair with about $10 \%$.

\footnotetext{
${ }^{4}$ As will be shown shortly, $\mathrm{W} / \mathrm{Z}$ gauge boson masses increase the branching fraction into a photon pair by about $10 \%$. We also note that the DM annihilation cross section into a photon pair depends on the velocity of dark matter, so only vector dark matter can explain Fermi-LAT line as will be shown later.
} 

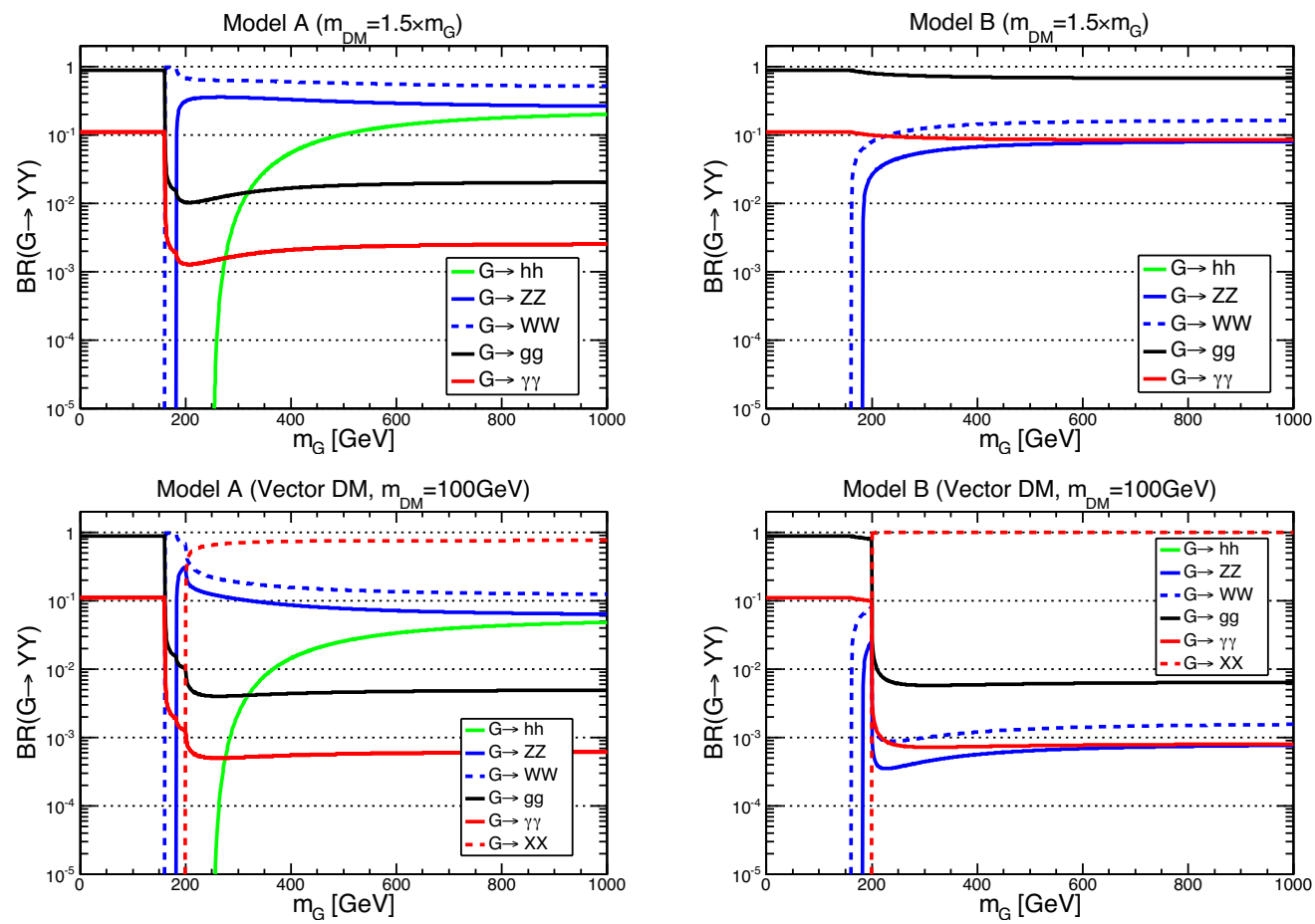

Figure 2. Branching fractions of decay rates of the KK graviton for Model A (left) and B (right), without (upper) or with (lower) the decay mode into a vector dark matter pair for $m_{\mathrm{DM}}=100 \mathrm{GeV}$. Green, blue(solid and dashed), black and red lines correspond to branching fractions into $h h, Z Z, W W, g g, \gamma \gamma$, respectively. We have taken $c_{X}=1, c_{V}=c_{g}=c_{\gamma}=0.03$ in common, and $c_{H}=1$ in Model A (left) and $c_{H}=0$ in Model B (right).

Above the $W W$ threshold, the decay modes into $W W, Z Z$ are dominant in Model A, while they are comparable to the one into a photon pair in Model B. On the other hand, if the KK graviton is allowed to decay into a dark matter pair, the extra decay mode becomes dominant and the branching fractions of the other decay modes scale down accordingly. In the lower panel of figure 2, the branching fractions of KK graviton decay modes for vector dark matter with $m_{\mathrm{DM}}=100 \mathrm{GeV}$ are shown. For dark matter with another spin, the extra decay mode into a dark matter pair shows a qualitatively similar behaviour. We note that the branching fractions of the KK graviton decay also depend on the presence of the decay mode into a top quark pair as shown in ref. [47].

We note that in Model B, if gluons are localized on the UV brane too, then $c_{W}^{G}=$ $c_{B}^{G} \simeq 0.03$ and $c_{\psi}^{G}, c_{H}^{G}, c_{g}^{G} \ll c_{W, B}^{G}$. Then, for universal electroweak gauge couplings with $c_{W}^{G}=c_{B}^{G}$, the branching fraction of the $\gamma \gamma$ decay mode becomes about

$$
\frac{\Gamma_{G}(\gamma \gamma)}{\Gamma_{G}(\text { total })} \simeq \frac{\left(c_{B}^{G}\right)^{2}}{3\left(c_{W}^{G}\right)^{2}+\left(c_{B}^{G}\right)^{2}}=0.25 .
$$

Thus, in this case, the branching fraction of the DM annihilation into a photon pair would be too large to explain the Fermi gamma-ray line. However, as will be discussed, if there are extra annihilation channels coming from radion mediation, it is possible to reduce the 


\begin{tabular}{|c|l|c|c|c|}
\hline channels & DM mass & $\mathrm{X}(\mathrm{s}=0)$ & $\mathrm{X}(\mathrm{s}=1 / 2)$ & $\mathrm{X}(\mathrm{s}=1)$ \\
\hline s-channel & $m_{\mathrm{DM}}<m_{W}$ & d-wave & p-wave & s-wave \\
\hline s-channel & $m_{\mathrm{DM}}>m_{W}$ & s-wave & p-wave & s-wave \\
\hline t-channel & $m_{\mathrm{DM}}>m_{G}$ & s-wave & s-wave & s-wave \\
\hline
\end{tabular}

Table 1. Suppression in Dark Matter annihilation cross sections with graviton mediator, depending on the spin of Dark Matter.

branching fraction of the DM annihilation into a photon pair, being consistent with the Fermi gamma-ray line.

Henceforth, we focus on the graviton mediator for dark matter annihilations and denote the KK graviton couplings simply by $c_{i}$ with $i$ running over the SM particles. Before going into the details of each dark matter of a given spin, we summarize the suppression of dark matter annihilation cross sections in table 1 . We note that the $W W / Z Z$ s-channels in Model $\mathrm{B}$ are roughly given by those in Model $\mathrm{A}$ with $c_{H}$ being replaced by a volumesuppressed quantity, $c_{V}$.

\subsection{Scalar dark matter}

In Model $\mathrm{A}$, for $c_{V} \ll c_{H}$, we get the annihilation cross section of scalar dark matter going into a pair of massive gauge bosons

$$
\begin{aligned}
(\sigma v)_{S S \rightarrow Z Z} & \simeq \frac{3 c_{S}^{2} c_{H}^{2}}{16 \pi \Lambda^{4}} \frac{m_{S}^{2} m_{Z}^{4}}{\left(4 m_{S}^{2}-m_{G}^{2}\right)^{2}+\Gamma_{G}^{2} m_{G}^{2}}\left(1-\frac{4 m_{S}^{2}}{m_{G}^{2}}\right)^{2}\left(1-\frac{m_{Z}^{2}}{m_{S}^{2}}\right)^{\frac{1}{2}}, \\
(\sigma v)_{S S \rightarrow W W} & \simeq \frac{3 c_{S}^{2} c_{H}^{2}}{8 \pi \Lambda^{4}} \frac{m_{S}^{2} m_{W}^{4}}{\left(4 m_{S}^{2}-m_{G}^{2}\right)^{2}+\Gamma_{G}^{2} m_{G}^{2}}\left(1-\frac{4 m_{S}^{2}}{m_{G}^{2}}\right)^{2}\left(1-\frac{m_{Z}^{2}}{m_{S}^{2}}\right)^{\frac{1}{2}}
\end{aligned}
$$

where the decay width of the KK graviton is given by

$$
\Gamma_{G}=\Gamma(h h)+\Gamma(Z Z)+\Gamma(W W)+\Gamma(g g)+\Gamma(S S)
$$

with

$$
\begin{aligned}
\Gamma(h h) & =\frac{c_{H}^{2} m_{G}^{3}}{960 \pi \Lambda^{2}}\left(1-\frac{4 m_{h}^{2}}{m_{G}^{2}}\right)^{\frac{5}{2}} \\
\Gamma(Z Z) & =\frac{c_{V}^{2} m_{G}^{3}}{960 \pi \Lambda^{2}}\left(1-\frac{4 m_{Z}^{2}}{m_{G}^{2}}\right)^{\frac{1}{2}}\left(13+\frac{56 m_{Z}^{2}}{m_{G}^{2}}+\frac{48 m_{Z}^{4}}{m_{G}^{4}}\right), \\
\Gamma(W W) & =\frac{c_{V}^{2} m_{G}^{3}}{480 \pi \Lambda^{2}}\left(1-\frac{4 m_{W}^{2}}{m_{G}^{2}}\right)^{\frac{1}{2}}\left(13+\frac{56 m_{W}^{2}}{m_{G}^{2}}+\frac{48 m_{W}^{4}}{m_{G}^{4}}\right), \\
\Gamma(\gamma \gamma) & =\frac{c_{\gamma}^{2} m_{G}^{3}}{80 \pi \Lambda^{2}} \\
\Gamma(g g) & =\frac{c_{g}^{2} m_{G}^{3}}{10 \pi \Lambda^{2}} \\
\Gamma(S S) & =\frac{c_{S}^{2} m_{G}^{3}}{960 \pi \Lambda^{2}}\left(1-\frac{4 m_{S}^{2}}{m_{G}^{2}}\right)^{\frac{5}{2}} .
\end{aligned}
$$


Moreover, the annihilation cross section into a Higgs pair is d-wave suppressed and it is given by

$$
(\sigma v)_{S S \rightarrow h h} \simeq v^{4} \cdot \frac{c_{S}^{2} c_{H}^{2}}{720 \pi \Lambda^{4}} \frac{m_{S}^{6}}{\left(4 m_{S}^{2}-m_{G}^{2}\right)^{2}+\Gamma_{G}^{2} m_{G}^{2}}\left(1-\frac{m_{h}^{2}}{m_{S}^{2}}\right)^{\frac{5}{2}} .
$$

In Model $\mathrm{B}$, taking $c_{H} \ll c_{V}$, the corresponding annihilation cross sections for scalar dark matter are [47]

$$
\begin{aligned}
(\sigma v)_{S S \rightarrow Z Z} & \simeq \frac{3 c_{S}^{2} c_{V}^{2}}{16 \pi \Lambda^{4}} \frac{m_{S}^{2} m_{Z}^{4}}{\left(4 m_{S}^{2}-m_{G}^{2}\right)^{2}+\Gamma_{G}^{2} m_{G}^{2}}\left(1-\frac{4 m_{S}^{2}}{m_{G}^{2}}\right)^{2}\left(1-\frac{m_{Z}^{2}}{m_{S}^{2}}\right)^{\frac{1}{2}} \\
(\sigma v)_{S S \rightarrow W W} & \simeq \frac{3 c_{S}^{2} c_{V}^{2}}{8 \pi \Lambda^{4}} \frac{m_{S}^{2} m_{W}^{4}}{\left(4 m_{S}^{2}-m_{G}^{2}\right)^{2}+\Gamma_{G}^{2} m_{G}^{2}}\left(1-\frac{4 m_{S}^{2}}{m_{G}^{2}}\right)^{2}\left(1-\frac{m_{Z}^{2}}{m_{S}^{2}}\right)^{\frac{1}{2}} .
\end{aligned}
$$

For both models, we find that the annihilation cross sections into a photon pair or a gluon pair are always d-wave and are given [47] by

$$
\begin{aligned}
& (\sigma v)_{S S \rightarrow \gamma \gamma} \simeq v^{4} \cdot \frac{c_{S}^{2} c_{\gamma}^{2}}{60 \pi \Lambda^{4}} \frac{m_{S}^{6}}{\left(4 m_{S}^{2}-m_{G}^{2}\right)^{2}+\Gamma_{G}^{2} m_{G}^{2}}, \\
& (\sigma v)_{S S \rightarrow g g} \simeq v^{4} \cdot \frac{2 c_{S}^{2} c_{g}^{2}}{15 \pi \Lambda^{4}} \frac{m_{S}^{6}}{\left(4 m_{S}^{2}-m_{G}^{2}\right)^{2}+\Gamma_{G}^{2} m_{G}^{2}} .
\end{aligned}
$$

In the case with heavy dark matter where the contributions of higher KK modes of graviton to the s-channel annihilations become important, we need to perform the following KK sum,

$$
\mathcal{S}(s)=\frac{1}{\Lambda^{2}} \sum_{n=1}^{\infty} \frac{1}{s-m_{n}^{2}+i m_{n} \Gamma_{n}} .
$$

Here, $\Gamma_{n}$ denotes the total width of the graviton with KK number $s$ and mass $m_{n}$ and is given by

$$
\Gamma_{n} \approx \eta m_{n}\left(\frac{m_{n}}{\Lambda}\right)^{2}, \quad \eta=\frac{c_{H}^{2}}{240 \pi} .
$$

The KK graviton masses are determined by the zeros of $J_{1}\left(x_{n}\right)$ as $m_{n}=x_{n} k \Lambda / M_{P}$, with $x_{1}=3.83$ and $x_{n}=\pi(n+1 / 4)+\mathcal{O}\left(n^{-1}\right)$. For $\eta s \ll \Lambda^{2}$, we replace the KK graviton propagator with the first KK graviton, $\Lambda^{-2} /\left(s-m_{1}^{2}+i m_{1} \Gamma_{1}\right)$, by the KK sum as follows [47],

$$
\mathcal{S}(s) \simeq-\frac{1}{4 \Lambda^{2} \sqrt{s}} \frac{x_{1}}{m_{1}} \frac{J_{2}(\sigma)}{J_{1}(\sigma)}
$$

with $\sigma \simeq\left(x_{1} \sqrt{s} / m_{1}\right)\left(1+i \eta s / 2 \Lambda^{2}\right)$. Henceforth we always take into account the higher KK modes of graviton for our discussion.

Finally, when $m_{S}>m_{G}$, there is an extra contribution to the annihilation cross section, due to the t-channel for both models, as follows,

$$
(\sigma v)_{S S \rightarrow G G} \simeq \frac{4 c_{S}^{4} m_{S}^{2}}{9 \pi \Lambda^{4}} \frac{\left(1-r_{S}\right)^{\frac{9}{2}}}{r_{S}^{4}\left(2-r_{S}\right)^{2}}
$$


with $r_{S}=\left(\frac{m_{G}}{m_{S}}\right)^{2}$. The t-channel annihilation cross section becomes singular for $r_{S} \ll 1$, which is a sign of unitarity violation in the case with the KK gravitons in the effective theory. The unitarity bound, $\sigma<1 / s \simeq 1 / m_{S}^{2}$, implies that $c_{S} m_{G} / \Lambda \lesssim(9 \pi v)^{1 / 4}\left(m_{G} / m_{S}\right)^{3}$ for a single KK graviton. On the other hand, each higher KK mode contribution is suppressed by $\left(m_{G} / m_{K K}\right)^{8}$ as compared to the first KK graviton contribution, occupying only less than $0.7 \%$ of the total t-channel cross section. Thus, we can ignore the higher KK mode contributions safely. We note that the t-channel annihilation cross sections for dark matter with another spin show a similar singular behavior.

In Model $\mathrm{A}$ and $\mathrm{B}$, the annihilation of scalar dark matter into $W W$ or $Z Z$ is s-wave, unlike the annihilation channels into massless gauge bosons. But, the corresponding annihilation cross sections are suppressed by $m_{Z, W}^{4} / m_{S}^{2}$, as compared to vector dark matter in the next section. Thus, for $m_{Z, W} \ll m_{S}$, the annihilation cross sections of scalar dark matter are much smaller than those of vector dark matter, for a fixed KK graviton coupling. If $m_{Z, W} \gtrsim m_{S}$, the annihilation cross section of scalar dark matter becomes a sizable s-wave.

\subsection{Fermion dark matter}

In Model $\mathrm{A}$, for $c_{H} \gg c_{V}$, the annihilation cross sections for a pair of massive gauge bosons are $[47]$

$$
\begin{aligned}
(\sigma v)_{\chi \bar{\chi} \rightarrow Z Z} \simeq & v^{2} \cdot \frac{c_{\chi}^{2} c_{H}^{2}}{144 \pi \Lambda^{4}} \frac{m_{\chi}^{6}}{\left(4 m_{\chi}^{2}-m_{G}^{2}\right)^{2}+\Gamma_{G}^{2} m_{G}^{2}} \\
& \times\left(1+\frac{3 m_{Z}^{2}}{m_{\chi}^{2}}+\frac{31}{8} \frac{m_{Z}^{4}}{m_{\chi}^{4}}-\frac{3 m_{Z}^{4}}{m_{G}^{2} m_{\chi}^{2}}+\frac{6 m_{Z}^{4}}{m_{G}^{4}}\right)\left(1-\frac{m_{Z}^{2}}{m_{\chi}^{2}}\right)^{\frac{1}{2}} \\
(\sigma v)_{\chi \bar{\chi} \rightarrow W W} \simeq & v^{2} \cdot \frac{c_{\chi}^{2} c_{H}^{2}}{72 \pi \Lambda^{4}} \frac{m_{\chi}^{6}}{\left(4 m_{\chi}^{2}-m_{G}^{2}\right)^{2}+\Gamma_{G}^{2} m_{G}^{2}} \\
& \left(1+\frac{3 m_{W}^{2}}{m_{\chi}^{2}}+\frac{31}{8} \frac{m_{W}^{4}}{m_{\chi}^{4}}-\frac{3 m_{W}^{4}}{m_{G}^{2} m_{\chi}^{2}}+\frac{6 m_{W}^{4}}{m_{G}^{4}}\right)\left(1-\frac{m_{W}^{2}}{m_{\chi}^{2}}\right)^{\frac{1}{2}}
\end{aligned}
$$

where the decay rate of the KK graviton is

$$
\Gamma_{G}=\Gamma(h h)+\Gamma(Z Z)+\Gamma(W W)+\Gamma(g g)+\Gamma(\chi \bar{\chi})
$$

with

$$
\Gamma(\chi \bar{\chi})=\frac{c_{\chi}^{2} m_{G}}{160 \pi}\left(\frac{m_{G}}{\Lambda}\right)^{2}\left(1-\frac{4 m_{\chi}^{2}}{m_{G}^{2}}\right)^{\frac{3}{2}}\left(1+\frac{8}{3} \frac{m_{\chi}^{2}}{m_{G}^{2}}\right) .
$$

Moreover, the annihilation cross section into a Higgs pair is p-wave suppressed and it is given by

$$
(\sigma v)_{\chi \bar{\chi} \rightarrow h h} \simeq v^{2} \cdot \frac{c_{\chi}^{2} c_{H}^{2}}{144 \pi \Lambda^{4}} \frac{m_{\chi}^{6}}{\left(4 m_{\chi}^{2}-m_{G}^{2}\right)^{2}+\Gamma_{G}^{2} m_{G}^{2}}\left(1-\frac{m_{h}^{2}}{m_{\chi}^{2}}\right)^{\frac{5}{2}}
$$


On the other hand, in Model $\mathrm{B}$, for $c_{H} \ll c_{V}$, the corresponding annihilation cross sections for fermion dark matter are [47]

$$
\begin{aligned}
(\sigma v)_{\chi \bar{\chi} \rightarrow Z Z} \simeq & v^{2} \cdot \frac{c_{\chi}^{2} c_{V}^{2}}{144 \pi \Lambda^{4}} \frac{m_{\chi}^{6}}{\left(m_{G}^{2}-4 m_{\chi}^{2}\right)^{2}+\Gamma_{G}^{2} m_{G}^{2}}\left(1-\frac{m_{Z}^{2}}{m_{\chi}^{2}}\right)^{\frac{1}{2}} \\
& \times\left(12-\frac{9 m_{Z}^{2}}{m_{\chi}^{2}}+\frac{39 m_{Z}^{4}}{8 m_{\chi}^{4}}-\frac{3 m_{Z}^{4}}{m_{G}^{2} m_{\chi}^{2}}+\frac{6 m_{Z}^{4}}{m_{G}^{4}}\right) \\
(\sigma v)_{\chi \bar{\chi} \rightarrow W W} \simeq & v^{2} \cdot \frac{c_{\chi}^{2} c_{V}^{2}}{72 \pi \Lambda^{4}} \frac{m_{\chi}^{6}}{\left(m_{G}^{2}-4 m_{\chi}^{2}\right)^{2}+\Gamma_{G}^{2} m_{G}^{2}}\left(1-\frac{m_{W}^{2}}{m_{\chi}^{2}}\right)^{\frac{1}{2}} \\
& \left(12-\frac{9 m_{W}^{2}}{m_{\chi}^{2}}+\frac{39 m_{W}^{4}}{8 m_{\chi}^{4}}-\frac{3 m_{W}^{4}}{m_{G}^{2} m_{\chi}^{2}}+\frac{6 m_{W}^{4}}{m_{G}^{4}}\right)
\end{aligned}
$$

For both models, we obtain the annihilation cross sections into a pair of massless gauge bosons [47] as

$$
\begin{aligned}
& (\sigma v)_{\chi \bar{\chi} \rightarrow \gamma \gamma} \simeq v^{2} \cdot \frac{c_{\chi}^{2} c_{\gamma}^{2}}{12 \pi \Lambda^{4}} \frac{m_{\chi}^{6}}{\left(4 m_{\chi}^{2}-m_{G}^{2}\right)^{2}+\Gamma_{G}^{2} m_{G}^{2}} \\
& (\sigma v)_{\chi \bar{\chi} \rightarrow g g} \simeq v^{2} \cdot \frac{2 c_{\chi}^{2} c_{g}^{2}}{3 \pi \Lambda^{4}} \frac{m_{\chi}^{6}}{\left(4 m_{\chi}^{2}-m_{G}^{2}\right)^{2}+\Gamma_{G}^{2} m_{G}^{2}} .
\end{aligned}
$$

On the other hand, when $m_{\chi}>m_{G}$, there is an extra contribution to the annihilation cross section, due to the t-channel for both models, as follows,

$$
(\sigma v)_{\chi \bar{\chi} \rightarrow G G} \simeq \frac{c_{\chi}^{4} m_{\chi}^{2}}{16 \pi \Lambda^{4}} \frac{\left(1-r_{\chi}\right)^{\frac{7}{2}}}{r_{\chi}^{4}\left(2-r_{\chi}\right)^{2}}
$$

with $r_{\chi}=\left(\frac{m_{G}}{m_{\chi}}\right)^{2}$.

Consequently, in Model $\mathrm{A}$ and $\mathrm{B}$, for $m_{G}>m_{\chi}$, the annihilation cross sections of fermion dark matter are p-wave suppressed. On the other hand, for $m_{G}<m_{\chi}$, the t-channel annihilation into a pair of the KK gravitons is s-wave and becomes dominant in determine the relic density. As shown in figure 3 , for $m_{G}<m_{\chi}$, scalar or fermion dark matter can account for the relic density for small values of the effective KK graviton couplings. On the other hand, for $m_{G}>m_{\chi}$, it is the s-channel annihilations that determine the relic density, so the necessary KK graviton couplings should be larger than in the case with $m_{G}<m_{\chi}$. If radion mediation is included, of course, the necessary effective KK graviton coupling can be smaller for the relic density in both cases [47]. 


\subsection{Vector dark matter}

In Model $\mathrm{A}$, for $c_{V} \ll c_{H}$, the annihilation cross sections of vector dark matter going into a pair of massive gauge bosons are

$$
\begin{aligned}
(\sigma v)_{X X \rightarrow Z Z} \simeq & \frac{2 c_{X}^{2} c_{H}^{2}}{27 \pi \Lambda^{4}} \frac{m_{X}^{6}}{\left(m_{G}^{2}-4 m_{X}^{2}\right)^{2}+\Gamma_{G}^{2} m_{G}^{2}} \\
& \times\left(1+\frac{3 m_{Z}^{2}}{m_{X}^{2}}+\frac{115}{32} \frac{m_{Z}^{4}}{m_{X}^{4}}-\frac{3}{4} \frac{m_{Z}^{4}}{m_{G}^{2} m_{X}^{2}}+\frac{3}{2} \frac{m_{Z}^{4}}{m_{G}^{4}}\right)\left(1-\frac{m_{Z}^{2}}{m_{X}^{2}}\right)^{\frac{1}{2}} \\
(\sigma v)_{X X \rightarrow W W} \simeq & \frac{4 c_{X}^{2} c_{H}^{2}}{27 \pi \Lambda^{4}} \frac{m_{X}^{6}}{\left(m_{G}^{2}-4 m_{X}^{2}\right)^{2}+\Gamma_{G}^{2} m_{G}^{2}} \\
& \times\left(1+\frac{3 m_{W}^{2}}{m_{X}^{2}}+\frac{115}{32} \frac{m_{W}^{4}}{m_{X}^{4}}-\frac{3}{4} \frac{m_{W}^{4}}{m_{G}^{2} m_{X}^{2}}+\frac{3}{2} \frac{m_{W}^{4}}{m_{G}^{4}}\right)\left(1-\frac{m_{W}^{2}}{m_{X}^{2}}\right)^{\frac{1}{2}}
\end{aligned}
$$

where the decay width of the KK graviton is

$$
\Gamma_{G}=\Gamma(h h)+\Gamma(Z Z)+\Gamma(W W)+\Gamma(g g)+\Gamma(X X)
$$

with

$$
\Gamma(X X)=\frac{c_{X}^{2} m_{G}^{3}}{960 \pi \Lambda^{2}}\left(1-\frac{4 m_{X}^{2}}{m_{G}^{2}}\right)^{\frac{1}{2}}\left(13+\frac{56 m_{X}^{2}}{m_{G}^{2}}+\frac{48 m_{X}^{4}}{m_{G}^{4}}\right) .
$$

Moreover, the annihilation cross section into a Higgs pair is s-wave and is given by

$$
(\sigma v)_{X X \rightarrow h h} \simeq \frac{2 c_{X}^{2} c_{H}^{2}}{27 \pi \Lambda^{4}} \frac{m_{X}^{6}}{\left(4 m_{X}^{2}-m_{G}^{2}\right)^{2}+\Gamma_{G}^{2} m_{G}^{2}}\left(1-\frac{m_{h}^{2}}{m_{X}^{2}}\right)^{\frac{5}{2}} .
$$

On the other hand, in Model B, $c_{H} \ll c_{V}$, the corresponding annihilation cross sections for vector dark matter are [47]

$$
\begin{aligned}
(\sigma v)_{X X \rightarrow Z Z} \simeq & \frac{2 c_{X}^{2} c_{V}^{2}}{27 \pi \Lambda^{4}} \frac{m_{X}^{6}}{\left(m_{G}^{2}-4 m_{X}^{2}\right)^{2}+\Gamma_{G}^{2} m_{G}^{2}}\left(1-\frac{m_{Z}^{2}}{m_{X}^{2}}\right)^{\frac{1}{2}} \\
& \times\left(12-\frac{9 m_{Z}^{2}}{m_{X}^{2}}+\frac{147}{32} \frac{m_{Z}^{4}}{m_{X}^{4}}-\frac{3}{4} \frac{m_{Z}^{4}}{m_{G}^{2} m_{X}^{2}}+\frac{3}{2} \frac{m_{Z}^{4}}{m_{G}^{4}}\right), \\
(\sigma v)_{X X \rightarrow W W} \simeq & \frac{4 c_{X}^{2} c_{V}^{2}}{27 \pi \Lambda^{4}} \frac{m_{X}^{6}}{\left(m_{G}^{2}-4 m_{X}^{2}\right)^{2}+\Gamma_{G}^{2} m_{G}^{2}}\left(1-\frac{m_{W}^{2}}{m_{X}^{2}}\right)^{\frac{1}{2}} \\
& \times\left(12-\frac{9 m_{W}^{2}}{m_{X}^{2}}+\frac{147}{32} \frac{m_{W}^{4}}{m_{X}^{4}}-\frac{3}{4} \frac{m_{W}^{4}}{m_{G}^{2} m_{X}^{2}}+\frac{3}{2} \frac{m_{W}^{4}}{m_{G}^{4}}\right) .
\end{aligned}
$$

For both models, we also obtain the annihilation cross sections into a pair of massless gauge bosons [47] as

$$
\begin{aligned}
(\sigma v)_{X X \rightarrow \gamma \gamma} & =\frac{8 c_{X}^{2} c_{\gamma}^{2}}{9 \pi \Lambda^{4}} \frac{m_{X}^{6}}{\left(4 m_{X}^{2}-m_{G}^{2}\right)^{2}+\Gamma_{G}^{2} m_{G}^{2}}, \\
(\sigma v)_{X X \rightarrow g g} & =\frac{64 c_{X}^{2} c_{g}^{2}}{9 \pi \Lambda^{4}} \frac{m_{X}^{6}}{\left(4 m_{X}^{2}-m_{G}^{2}\right)^{2}+\Gamma_{G}^{2} m_{G}^{2}} .
\end{aligned}
$$



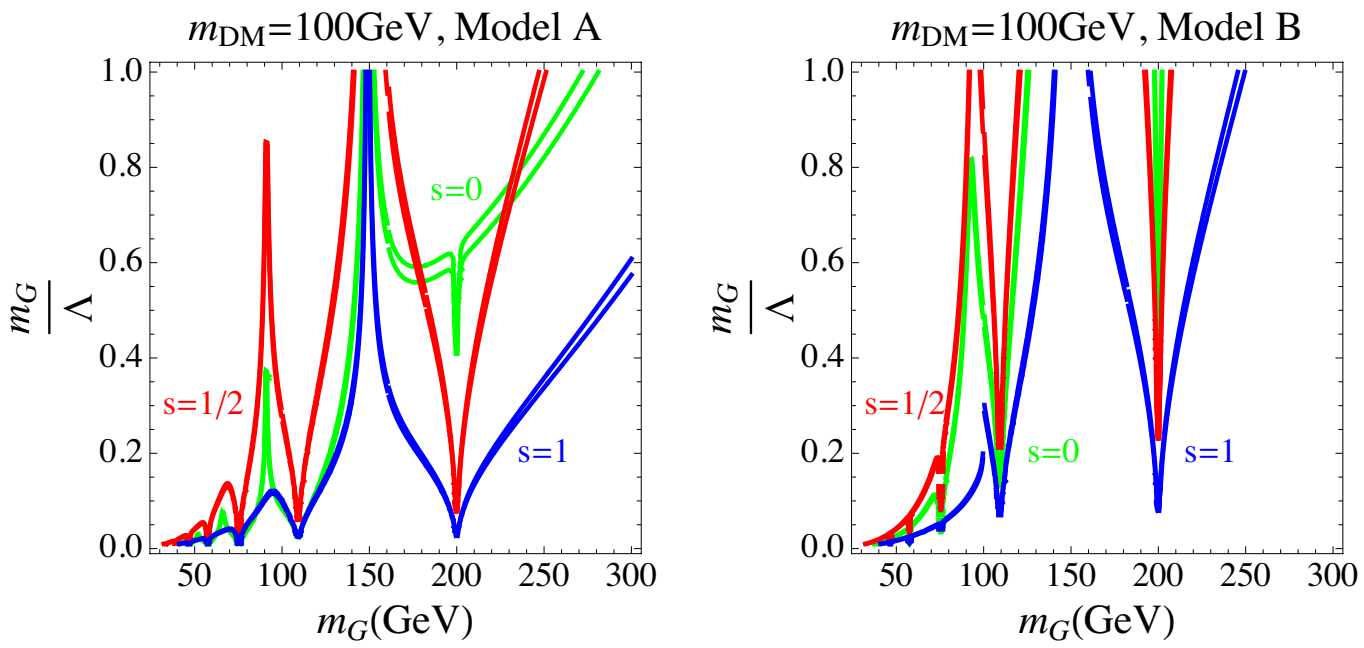

Figure 3. Parameter space of the effective DM coupling, $m_{G} / \Lambda$, vs $m_{G}$ for scalar (green), fermion (red) and vector (blue) dark matters, satisfying the relic density condition. We have taken $c_{X}=1$, $c_{V}=c_{g}=c_{\gamma}=0.03$ in common, and $c_{H}=1$ on left (Model A) and $c_{H}=0$ on right (Model B). Here, we note that spikes appearing for $m_{G}<2 m_{\mathrm{DM}}$ correspond to resonances due to the higher KK modes of graviton.

On the other hand, when $m_{X}>m_{G}$, there is an extra contribution to the annihilation cross section, due to the t-channel in both models, as follows,

$$
\begin{aligned}
(\sigma v)_{X X \rightarrow G G} \simeq & \frac{c_{X}^{4} m_{X}^{2}}{324 \pi \Lambda^{4}} \frac{\sqrt{1-r_{X}}}{r_{X}^{4}\left(2-r_{X}\right)^{2}}\left(176+192 r_{X}+1404 r_{X}^{2}-3108 r_{X}^{3}\right. \\
& \left.+1105 r_{X}^{4}+362 r_{X}^{5}+34 r_{X}^{6}\right)
\end{aligned}
$$

with $r_{X}=\left(\frac{m_{G}}{m_{X}}\right)^{2}$. Therefore, the total annihilation cross sections for vector dark matter are s-wave. Furthermore, the partial annihilation cross section into a photon pair is sizable due to the universal gravity couplings to gauge bosons, $c_{V}=c_{\gamma}=c_{g}$.

In figure 3, we have shown the parameter space of the KK graviton coupling vs the KK graviton mass for a fixed mass of vector dark mass, in comparison to the cases of scalar and fermion dark matters. We note that the annihilation cross section of vector dark matter is s-wave independent of dark matter mass, as compared to the cases of scalar and fermion dark matters. There, vector dark matter is strongly subject to the gamma-ray detection experiments at present, as will be shown later.

\subsection{Impact of Higgs portal couplings}

In this section, we discuss the effect of a Higgs portal coupling on our analysis of the DM annihilation cross sections and the direct detection of dark matter. In the case of Model A, where the Higgs doublet and dark matter are localized on the same IR brane, a renormalizable Higgs portal coupling to the scalar dark matter, $\mathcal{L} \supset-\lambda_{h S} H^{\dagger} H S^{2} / 4$, is allowed by any symmetry of our model setup. Non-renormalizable Higgs portal couplings to singlet fermion or vector dark matter can be written too but they depend on a UV 


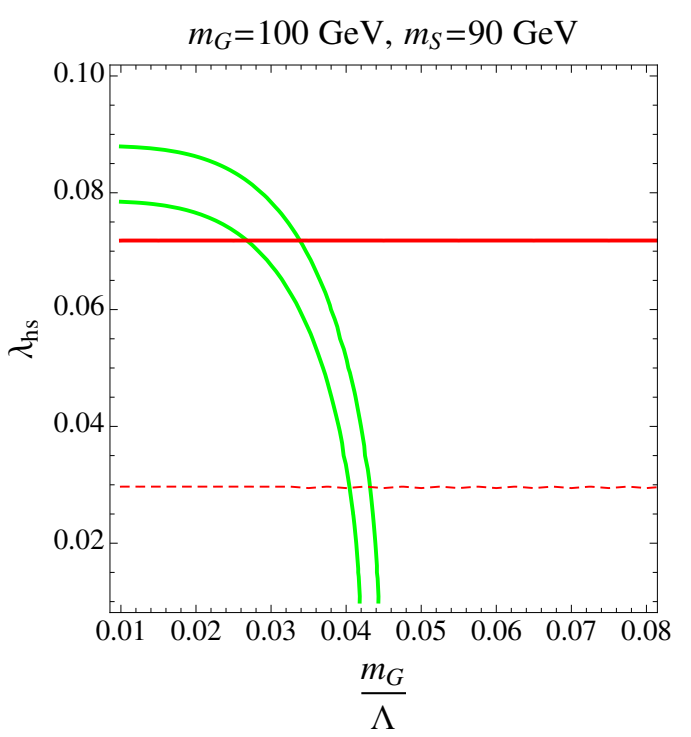

Figure 4. The KK graviton coupling versus Higgs portal coupling for scalar dark matter. The Planck $5 \sigma$ band for the relic density is bounded by green lines while the spin-independent DMnucleon scattering cross section, $\sigma_{S-N}=10^{-9} \mathrm{pb}$, for the minimum and maximum values of Higgsnucleon couplings, are shown in red solid and dashed lines, respectively [80].

completion. So, we focus on the case of scalar dark matter. In the case of Model B, there is no Higgs portal coupling at tree level, because the Higgs doublet and dark matter are localized on different branes. Instead, an effective quadratic Higgs coupling to dark matter is generated by the bulk graviton mediators, but there is no linear Higgs coupling to dark matter due to the fact that the KK graviton couples to the energy-momentum tensor.

There are three main effects of the Higgs portal coupling to scalar dark matter. First, it contributes to the DM annihilation cross section with extra s-channels of Higgs mediator. Second, when scalar dark matter is lighter than half the Higgs mass, the Higgs decay rates are affected by the invisible decay of Higgs into a pair of dark matter. From the search for the invisible decay of Higgs at the LHC, the branching fraction of Higgs invisible decay is constrained to $\operatorname{Br}(h \rightarrow S S)<0.65$ at $95 \%$ C.L. [81, 82]. For such a light dark matter below the WW threshold, the s-channel DM annihilations with KK graviton mediator are velocity-suppressed, so the extra annihilation channels with Higgs mediator can be dominant, unless the t-channel annihilation into a pair of the KK graviton is open. Third, the Higgs portal coupling could lead to a sizable spin-independent cross section between dark matter and nucleons so it is strongly constrained by the direct detection experiments $[83,84]$, in particular, below the WW threshold.

In figure 4, focusing on the region above the WW threshold, we depict the parameter space for the KK graviton coupling, $m_{G} / \Lambda$, vs the Higgs portal coupling $\lambda_{h S}$, satisfying the relic density with direct detection constraints. Whether the t-channel exchange is open or closed (as in the choice of masses in figure 4) the qualitative behaviour is the same. We note that the correct relic density can be obtained from a combination of the KK graviton coupling and the Higgs portal coupling, being consistent with the direct detection experiments. 


\subsection{The Fermi-LAT line}

We are in a position to discuss briefly the possibility of obtaining the Fermi gamma-ray line in the models. Most dark matter models explaining the Fermi gamma-ray line require new charged and/or neutral mediators [6-28] and invoke a strong coupling or a resonance in order to explain a large annihilation cross section into a photon pair for Fermi gamma-ray line at around $130 \mathrm{GeV}$. While couplings of dark matter or mediator field to a photon pair depend on unknown new charged particles in most cases in the literature, in our gravitymediated dark matter models, the couplings of the KK graviton mediator to a photon pair depend on the bulk profiles of KK gravitons and a photon.

For scalar dark matter, the partial annihilation cross section into a photon pair is dwave suppressed, so scalar dark matter does not give rise to a sizable branching fraction of monochromatic photons for the Fermi gamma-ray line. Likewise, for fermion dark matter, the annihilation cross section into a photon pair is p-wave suppressed at present, so fermion dark matter with graviton mediator is not relevant for the Fermi gamma-ray line either. For both scalar and fermion dark matter, extra annihilation channels coming from radion mediation [47] could reduce the branching fraction of monochromatic photons further, because the photon channel with radion mediation is loop-suppressed.

Unlike the previous cases, vector dark matter can accommodate the Fermi gamma-ray line in some cases. First, in Model A, the branching fraction for the DM annihilation into a photon pair is very small, due to large annihilation cross section into a pair of gauge bosons. Thus, we could not obtain the Fermi gamma-ray line in this case and there is no gamma-ray constraint for monochromatic photons. On the other hand, in Model B, when the t-channel annihilation into a pair of KK gravitons is forbidden kinematically for $m_{G}>m_{X}$, the branching fraction of the DM annihilation cross section into a photon pair can be sizable as the following,

$\frac{(\sigma v)_{\gamma \gamma}}{(\sigma v)_{\mathrm{tot}}} \simeq \frac{4}{9}\left\{4+\frac{1}{9}\left(4-3 \frac{m_{Z}^{2}}{m_{X}^{2}}+\frac{49}{32} \frac{m_{Z}^{4}}{m_{X}^{4}}-\frac{1}{4} \frac{m_{Z}^{4}}{m_{G}^{2} m_{X}^{2}}+\frac{1}{2} \frac{m_{Z}^{4}}{m_{G}^{4}}\right) \sqrt{1-\frac{m_{Z}^{2}}{m_{X}^{2}}}+2\left(m_{Z} \rightarrow m_{W}\right)\right\}^{-1}$.

Therefore, we find that taking vector dark matter mass to be $m_{X} \simeq 133 \mathrm{GeV}$ for Fermi gamma-ray line [5], the $W / Z$ mass dependence of the annihilation cross sections makes the branching fraction into a photon pair larger, with $(\sigma v)_{\gamma \gamma} /(\sigma v)_{\mathrm{KK}} \simeq 0.093(0.36)$ in Model $\mathrm{B}$ with $c_{g}=0.03\left(c_{g} \simeq 0\right)$. In figure 5 , we depict the parameter space accounting for the relic density with the KK graviton explaining the total relic density, $\langle\sigma v\rangle_{\mathrm{KK}}=\langle\sigma v\rangle_{\mathrm{th}}$, within $5 \sigma$ of the Planck data, $\Omega h^{2}=0.1199 \pm 0.0027$ [79], and the annihilation cross section into a photon pair necessary for the Fermi gamma-ray line [1,5]. To conclude, we find that for vector dark matter in Model B with all gauge bosons in bulk, both the relic density and the Fermi gamma-ray line are satisfied with the KK graviton mediator only, with a sizable effective KK graviton coupling, away from the resonance.

\section{Astrophysical constraints}

We consider astrophysical constraints on dark matter models with KK graviton mediator. Typical gamma-ray features are composed of monochromatic photons, continuum photons 


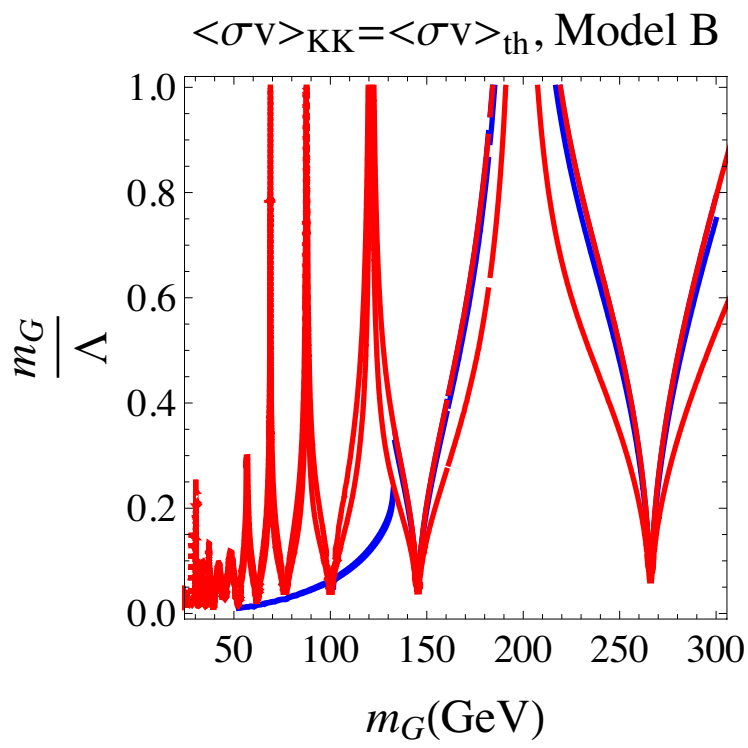

Figure 5. Parameter space of the effective DM coupling $m_{G} / \Lambda$ vs $m_{G}$ for vector dark matter, satisfying the relic density condition (blue) and the Fermi gamma-ray line at zero temperature (red). We have set $m_{X}=133 \mathrm{GeV}$ for Fermi gamma-ray line with $\langle\sigma v\rangle_{\gamma \gamma}=(0.67-3.16) \times 10^{-27} \mathrm{~cm}^{3} / \mathrm{s}[1]$ (within $1 \sigma$ range for NFW and Einasto dark matter profiles) and imposed the relic density within Planck $5 \sigma$ band. We have taken $c_{X}=1, c_{H}=c_{f}=0$ and $c_{V}=c_{\gamma}=c_{g}=0.03$.
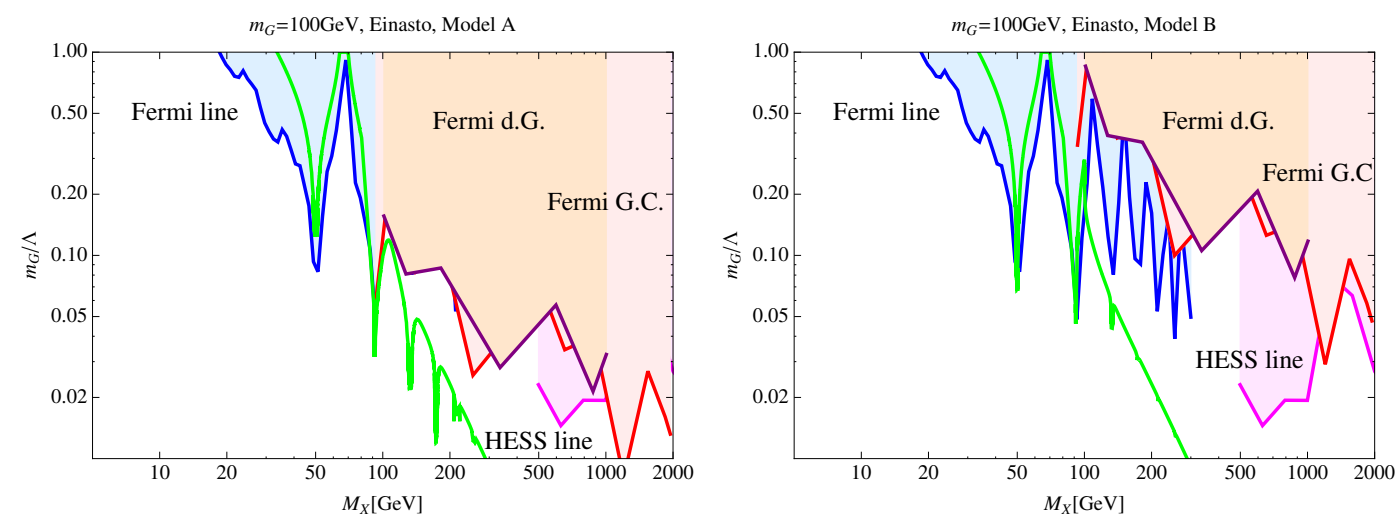

Figure 6. Astrophysical bounds on the parameter space, $M_{X}$ vs $m_{G} / \Lambda$, for vector dark matter. We have imposed the bounds from Fermi-LAT and HESS line searches, Fermi-LAT dwarf galaxies (d.G.) and Fermi-LAT galactic center (G.C.) on the annihilation cross section for Einasto dark matter profile. Green dashed lines show the Planck $5 \sigma$ band for the relic density. We have taken $c_{X}=1, c_{V}=c_{g}=c_{\gamma}=0.03$ in common, and $c_{H}=1$ on left (Model A) and $c_{H}=0$ on right (Model B).

and gamma-ray boxes. We impose gamma-ray constraints from Fermi-LAT and HESS on the model parameters. In figure 6 , we show the bounds from monochromatic and continuum photons on the $s$-channels of the models. In figure 7 , the gamma-ray constraints are given for the $t / u$-channels, which are responsible for the gamma-ray boxes. In both cases, we impose the relic density condition for the necessary total thermal cross section. 

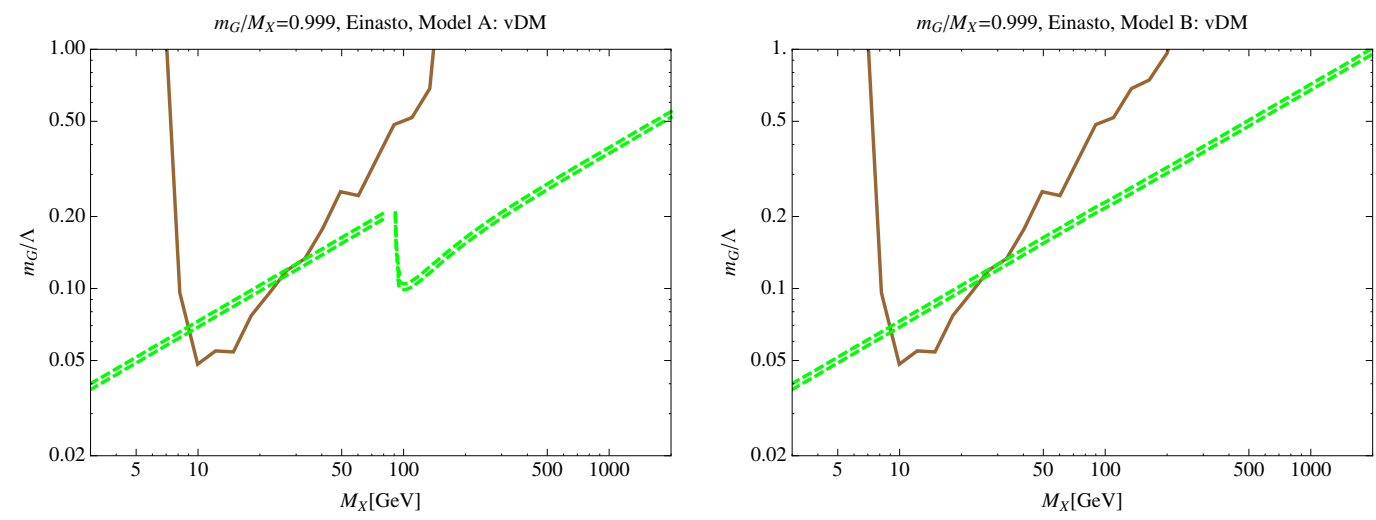

Figure 7. Bounds from narrow gamma-ray boxes for vector dark matter. We have imposed the bounds from Fermi-LAT galactic center (R16) on the annihilation cross section, $\langle\sigma v\rangle_{X X \rightarrow G G} \times$ $\operatorname{Br}(G \rightarrow \gamma \gamma)$, for Einasto dark matter profile [74]. Green dashed lines show the Planck $5 \sigma$ band for the relic density. We have taken $m_{G} / M_{X}=0.999, c_{X}=1, c_{V}=c_{g}=c_{\gamma}=0.03$ in common, and $c_{H}=1$ on left (Model A) and $c_{H}=0$ on right (Model B).

\subsection{Monochromatic photons}

In models with KK graviton mediator, dark matter annihilation into a photon pair leads to monochromatic photons at the energy of DM mass, so it can be constrained by gamma line searches at Fermi-LAT [5] and HESS [35]. From figure 6, for vector dark matter, it is shown that most of the parameter space below the $W W$ threshold explaining the relic density condition can be ruled out or in tension with the Fermi-LAT bounds, due to a sizable branching fraction of dark matter annihilation into a photon pair. For $M_{X}>m_{G}$, the Fermi-LAT bounds can be weakened due to the t-channel dominance. Nonetheless, a certain range of small KK graviton masses satisfying $M_{X}>m_{G}$ could be also ruled out by the gamma-ray box constraints as will be discussed later. On the other hand, there is no gamma-ray bound on scalar or fermion dark matter, as the annihilation cross sections into a photon pair are d-wave or p-wave suppressed.

\subsection{Continuum photons}

When DM annihilates into $\mathrm{W} / \mathrm{Z}$ gauge bosons or SM fermions, continuum photons can be generated from the secondary processes and they can be bounded by Fermi-LAT dwarf galaxies [36, 37], the gamma-ray from Fermi-LAT galactic center [43] and PAMLA antiproton data [44-46]. We consider the first two bounds as they are more stringent than or as strong as the last one. ${ }^{5}$ In figure 6 , for vector dark matter, we imposed the above bounds on the continuum photons in addition to Fermi-LAT and HESS monochromatic photons, as denoted in the figures, and found that the current HESS or continuum bounds are the strongest for heavy dark matter with $m_{X} \gtrsim m_{G}$, but they are compatible with

\footnotetext{
${ }^{5}$ For vector dark matter below WW threshold in Model A and for $m_{\mathrm{DM}}<m_{G}$ in Model B, the branching fraction of the $g g$ channel is about $90 \%$ the total annihilation cross section at present. The anti-proton bound on $\langle\sigma v\rangle_{g g}$ is about $10^{-26} \mathrm{~cm}^{3} / \mathrm{s}$ for MED or MIN propagation parameters [46], which is not as strong as the Fermi-LAT bound.
} 
the relic density condition. We note that for scalar dark matter, the $W W / Z Z$ channels, if kinematically open, are s-wave and dominant for $m_{X}<m_{G}$, so there is a similar strong bound from continuum photons, as for vector dark matter. On the other hand, for fermion dark matter, there is no bound from continuum photons.

\subsection{Gamma-ray boxes}

For the t-channel annihilation of dark matter into a pair of KK gravitons, the consequent decay of each KK graviton into a photon pair gives rise to a box-shaped gamma-ray spectrum [72-76]. Once the t-channel is open, it becomes dominant for determining the relic density. Thus, when the decay branching fraction of the KK graviton into a photon pair is sizable, it can be strongly constrained by Fermi-LAT and HESS gamma-ray constraints $[72,74]$.

In figure 7 , we first consider the bounds on narrow gamma-ray boxes and show the Fermi-LAT bounds (R16 for Einasto dark matter profile [5]) for vector dark matter models. Taking $m_{G} / M_{X}=0.999$ as an example, we find that vector dark matter masses of $9-30 \mathrm{GeV}$ have been ruled out by the Fermi-LAT bounds. On the other hand, in the case of scalar or fermion dark matter, there is no bound on narrow gamma-ray boxes, because the corresponding t-channel annihilation cross sections, (3.21) and (3.31), are highly suppressed for almost degenerate dark matter and KK graviton masses.

On the other hand, in figure 8, we show the Fermi-LAT bounds on wide gamma-ray boxes for scalar, fermion and vector dark matter in the models. In all the cases, it is shown that most of the parameter space being consistent with the relic density survives the FermiLAT gamma-ray constraints but the region with light dark matter around $5-15 \mathrm{GeV}$ has been excluded for $m_{G} / M_{X}=0.6$, due to the fact that dark matter annihilation cross section into a photon pair is sizable.

\section{Direct detection and collider bounds}

In this section, we comment on the direct detection and collider bounds on the models.

As discussed in the previous section, the interactions of dark matter to the light quarks are suppressed due to the geometric separation, but gluon interactions to dark matter can be sizable and dominant in determining the relic density for $m_{\mathrm{DM}}<m_{G}$. In this case, the corresponding gluon interactions are relevant for the direct detection of dark matter in underground experiments [46, 47]. For instance, even if the annihilation cross section of scalar dark matter into a gluon pair is velocity-suppressed, the DM-nucleon spin-independent scattering cross section is sizable [46, 47]. So, direct detection from XENON100 [83] or LUX [84] can constrain the parameter space with sizable KK graviton couplings at large DM masses ${ }^{6}$ complementing the gamma-ray constraints.

On the other hand, vector dark matter only can provide monochromatic photons that are compatible with Fermi gamma-ray line, when the KK graviton mediator is heavier than dark matter. In this case, we can obtain a sizable production cross section for the

\footnotetext{
${ }^{6}$ For instance, for $m_{G}=100 \mathrm{GeV}$, the strongest bound for scalar dark matter with $m_{S} \lesssim 300 \mathrm{GeV}$ is $m_{G} / \Lambda \lesssim 0.2$ [47]. But, spin-independent cross sections are suppressed for light dark matter masses, allowing for a sizable KK graviton coupling. More complete analysis will be done in a future work.
} 

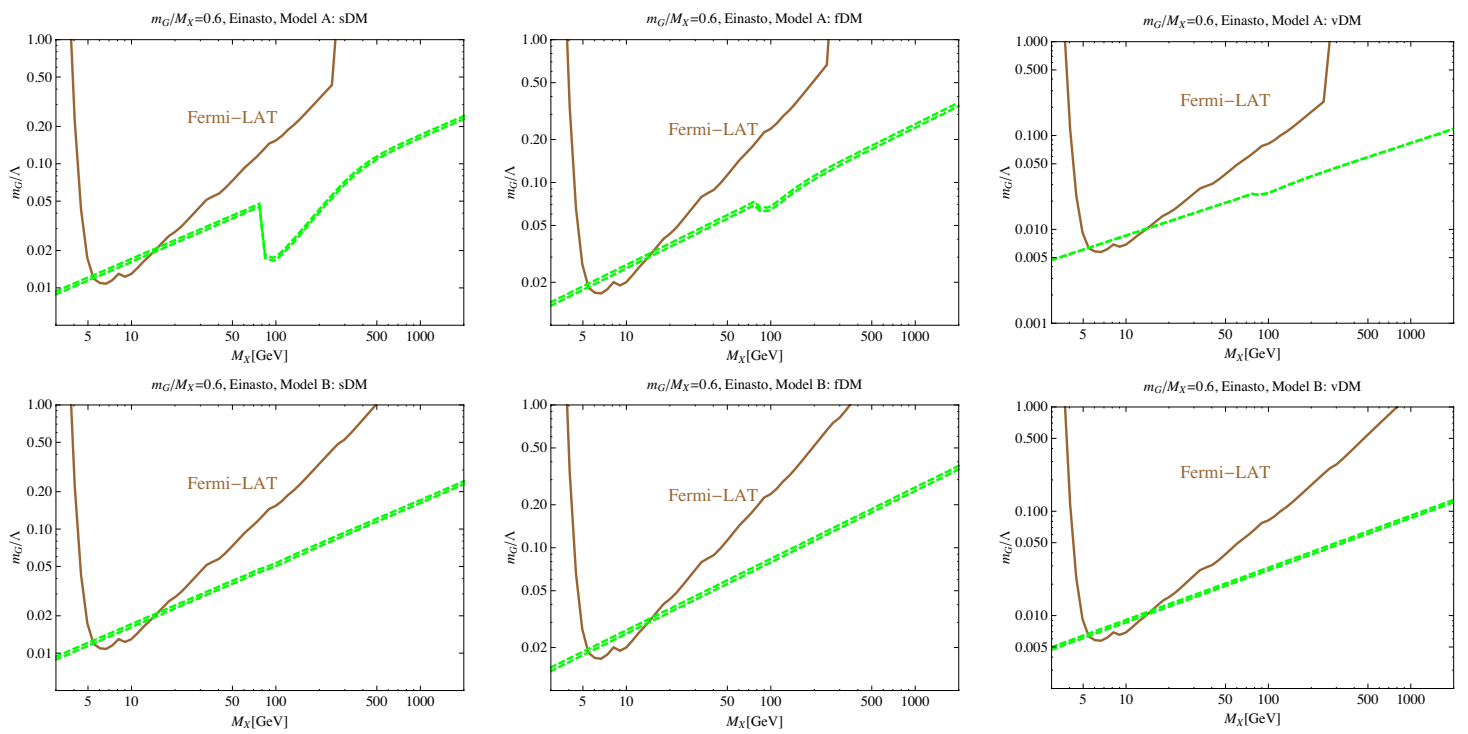

Figure 8. Bounds from wide gamma-ray boxes for scalar, fermion and vector dark matter, from left to right. We have imposed the bounds from Fermi-LAT galactic center (R16) on the annihilation cross section, $\langle\sigma v\rangle_{X X \rightarrow G G} \times \operatorname{Br}(G \rightarrow \gamma \gamma)$, for Einasto dark matter profile [74]. Green dashed lines show the Planck $5 \sigma$ band for the relic density. We have taken $m_{G} / M_{X}=0.6, c_{X}=1$, $c_{V}=c_{g}=c_{\gamma}=0.03$ in common, and $c_{H}=1$ in the upper panel (Model A) and $c_{H}=0$ in the lower panel (Model B).

KK graviton in association with a monophoton at the LHC [31, 85, 86] and its decay into a pair of dark matter may lead to a large missing energy. Then, we can impose the similar bounds on vector dark matter from monophoton searches [87, 88] as for axion or $Z^{\prime}$-mediated dark matter [29, 30]. Furthermore, if the KK graviton decay into a pair of dark matter is forbidden, we should rely on the resonant production of the KK graviton via gluon fusion into diphotons [89-91], or vector boson or photon fusion [92].

Other decays of the graviton, such as top or W,Z boson pairs, would be difficult to be performed at low mass, as the current resonant searches are based on boosted topologies. Decays to Higgs pairs have been studied in ref. [93], in a method which interpolates between the non-boosted and boosted regimes.

If the dominant decay of gravitons is to dark-matter pairs, searches using mono-photon and mono-jets in association with missing energy can be re-interpreted along the lines of ref. [94], with gluon fusion as the more likely production mechanism.

\section{Conclusions}

We have investigated a new dark matter model where gravity or composite sector such as a KK graviton in 5D warped spacetime or a spin-2 resonance in the dual conformal theory is responsible for the annihilation of dark matter into the SM particles. Dark matter annihilates mainly into gauge bosons, because the SM fermions and/or Higgs fields are separated from dark matter geometrically in the extra dimension. As a result, the KK 
graviton coupling to dark matter can allow for the correct relic density in the perturbative regime but it tends to be larger for scalar and fermion dark matters due to the velocitysuppressed annihilation cross section than for vector dark matter. We have shown that in the case of vector dark matter, the annihilation of dark matter into a photon pair can explain the Fermi gamma-ray line for $m_{X} \simeq 133 \mathrm{GeV} \lesssim m_{G}$.

Vector dark matter can be most strongly constrained by gamma-ray data, because the annihilation cross sections are s-wave and dark matter annihilates into photons with a sizable fraction. In this case, most of the parameter space below the $W W$ threshold being compatible with the relic density is in tension with the current gamma-ray constraints of Fermi-LAT line search in the galactic center. On the other hand, for $m_{X} \gtrsim m_{G}$, for which the correct relic density is obtained mainly by the annihilation of dark matter into a pair of the KK gravitons, most of the parameter space is consistent with the relic density and the current gamma-ray constraints, apart from the region with the light dark matter around $10 \mathrm{GeV}$. The scenarios of the spin-2 mediator can be tested further in various ways, by future indirect detection with gamma-ray rays, direct detection and the resonance production of the spin-2 mediator at the LHC.

\section{Acknowledgments}

HML would like to thank CERN Theory Group for its hospitality during his visits to CERN in Jan 2013 and Jan 2014 where the present work was initiated and finally finished. The work of HML is supported in part by Basic Science Research Program through the National Research Foundation of Korea(NRF) funded by the Ministry of Education, Science and Technology(2013R1A1A2007919). The work of MP is supported by a CERN-Korean fellowship and the World Premier International Research Center Initiative (WPI Initiative), MEXT, Japan. The work of VS is supported by the Science Technology and Facilities Council (STFC) under grant number ST/J000477/1.

Open Access. This article is distributed under the terms of the Creative Commons Attribution License (CC-BY 4.0), which permits any use, distribution and reproduction in any medium, provided the original author(s) and source are credited.

\section{References}

[1] C. Weniger, A Tentative Gamma-Ray Line from Dark Matter Annihilation at the Fermi Large Area Telescope, JCAP 08 (2012) 007 [arXiv: 1204.2797] [INSPIRE].

[2] E. Tempel, A. Hektor and M. Raidal, Fermi 130 GeV gamma-ray excess and dark matter annihilation in sub-haloes and in the Galactic centre, JCAP 09 (2012) 032 [Addendum ibid. 1211 (2012) A01] [arXiv:1205.1045] [INSPIRE].

[3] M. Su and D.P. Finkbeiner, Strong Evidence for Gamma-ray Line Emission from the Inner Galaxy, arXiv:1206.1616 [INSPIRE].

[4] D.P. Finkbeiner, M. Su and C. Weniger, Is the $130 \mathrm{GeV}$ Line Real? A Search for Systematics in the Fermi-LAT Data, JCAP 01 (2013) 029 [arXiv:1209.4562] [INSPIRE].

[5] Fermi-LAT collaboration, M. Ackermann et al., Search for Gamma-ray Spectral Lines with the Fermi Large Area Telescope and Dark Matter Implications, Phys. Rev. D 88 (2013) 082002 [arXiv: 1305.5597] [INSPIRE]. 
[6] T. Bringmann, X. Huang, A. Ibarra, S. Vogl and C. Weniger, Fermi LAT Search for Internal Bremsstrahlung Signatures from Dark Matter Annihilation, JCAP 07 (2012) 054 [arXiv:1203.1312] [INSPIRE].

[7] J.M. Cline, 130 GeV dark matter and the Fermi gamma-ray line, Phys. Rev. D 86 (2012) 015016 [arXiv: 1205.2688] [INSPIRE].

[8] E. Dudas, Y. Mambrini, S. Pokorski and A. Romagnoni, Extra U(1) as natural source of a monochromatic gamma ray line, JHEP 10 (2012) 123 [arXiv:1205.1520] [INSPIRE].

[9] K.-Y. Choi and O. Seto, A Dirac right-handed sneutrino dark matter and its signature in the gamma-ray lines, Phys. Rev. D 86 (2012) 043515 [Erratum ibid. D 86 (2012) 089904] [arXiv: 1205.3276] [INSPIRE].

[10] B. Kyae and J.-C. Park, 130 GeV Fermi gamma-ray line from dark matter decay, Phys. Lett. B 718 (2013) 1425 [arXiv: 1205.4151] [INSPIRE].

[11] A. Rajaraman, T.M.P. Tait and D. Whiteson, Two Lines or Not Two Lines? That is the Question of Gamma Ray Spectra, JCAP 09 (2012) 003 [arXiv: 1205.4723] [INSPIRE].

[12] B.S. Acharya, G. Kane, P. Kumar, R. Lu and B. Zheng, Mixed Wino-Axion Dark Matter in String/M Theory and the $130 \mathrm{GeV}$ Gamma-line 'Signal', arXiv:1205.5789 [INSPIRE].

[13] M.R. Buckley and D. Hooper, Implications of a $130 \mathrm{GeV}$ Gamma-Ray Line for Dark Matter, Phys. Rev. D 86 (2012) 043524 [arXiv:1205.6811] [INSPIRE].

[14] D. Das, U. Ellwanger and P. Mitropoulos, A $130 \mathrm{GeV}$ photon line from dark matter annihilation in the NMSSM, JCAP 08 (2012) 003 [arXiv:1206.2639] [INSPIRE].

[15] Z. Kang, T. Li, J. Li and Y. Liu, Brightening the (130 GeV) Gamma-Ray Line, arXiv:1206.2863 [INSPIRE].

[16] J.-C. Park and S.C. Park, Radiatively decaying scalar dark matter through U(1) mixings and the Fermi 130 GeV gamma-ray line, Phys. Lett. B 718 (2013) 1401 [arXiv:1207.4981] [INSPIRE].

[17] S. Tulin, H.-B. Yu and K.M. Zurek, Three Exceptions for Thermal Dark Matter with Enhanced Annihilation to $\gamma \gamma$, Phys. Rev. D 87 (2013) 036011 [arXiv:1208.0009] [INSPIRE].

[18] T. Li, J.A. Maxin, D.V. Nanopoulos and J.W. Walker, A 125.5 GeV Higgs Boson in F-SU(5): Imminently Observable Proton Decay, A 130 GeV Gamma-ray Line and SUSY Multijets \& Light Stops at the LHC8, Eur. Phys. J. C 72 (2012) 2246 [arXiv:1208.1999] [INSPIRE].

[19] J.M. Cline, A.R. Frey and G.D. Moore, Composite magnetic dark matter and the $130 \mathrm{GeV}$ line, Phys. Rev. D 86 (2012) 115013 [arXiv: 1208.2685] [INSPIRE].

[20] Y. Bai and J. Shelton, Gamma Lines without a Continuum: Thermal Models for the Fermi-LAT $130 \mathrm{GeV}$ Gamma Line, JHEP 12 (2012) 056 [arXiv:1208.4100] [INSPIRE].

[21] L. Bergstrom, The 130 GeV Fingerprint of Right-Handed Neutrino Dark Matter, Phys. Rev. D 86 (2012) 103514 [arXiv:1208.6082] [INSPIRE].

[22] L. Wang and X.-F. Han, $130 \mathrm{GeV}$ gamma-ray line and enhancement of $h \rightarrow \gamma \gamma$ in the Higgs triplet model plus a scalar dark matter, Phys. Rev. D 87 (2013) 015015 [arXiv:1209.0376] [INSPIRE].

[23] K. Schmidt-Hoberg, F. Staub and M.W. Winkler, Enhanced diphoton rates at Fermi and the LHC, JHEP 01 (2013) 124 [arXiv:1211.2835] [INSPIRE]. 
[24] Y. Farzan and A.R. Akbarieh, Natural explanation for $130 \mathrm{GeV}$ photon line within vector boson dark matter model, Phys. Lett. B 724 (2013) 84 [arXiv:1211.4685] [INSPIRE].

[25] G. Chalons, M.J. Dolan and C. McCabe, Neutralino dark matter and the Fermi gamma-ray lines, JCAP 02 (2013) 016 [arXiv:1211.5154] [INSPIRE].

[26] J. Fan and M. Reece, Probing Charged Matter Through Higgs Diphoton Decay, Gamma Ray Lines and EDMs, JHEP 06 (2013) 004 [arXiv:1301.2597] [INSPIRE].

[27] C.B. Jackson, G. Servant, G. Shaughnessy, T.M.P. Tait and M. Taoso, Gamma-ray lines and One-Loop Continuum from s-channel Dark Matter Annihilations, JCAP 07 (2013) 021 [arXiv: 1302.1802] [INSPIRE].

[28] K.-Y. Choi, H.M. Lee and O. Seto, Vector Higgs-portal dark matter and Fermi-LAT gamma ray line, Phys. Rev. D 87 (2013) 123541 [arXiv:1304.0966] [InSPIRE].

[29] H.M. Lee, M. Park and W.-I. Park, Fermi Gamma Ray Line at $130 \mathrm{GeV}$ from Axion-Mediated Dark Matter, Phys. Rev. D 86 (2012) 103502 [arXiv:1205.4675] [INSPIRE].

[30] H.M. Lee, M. Park and W.-I. Park, Axion-mediated dark matter and Higgs diphoton signal, JHEP 12 (2012) 037 [arXiv:1209.1955] [InSPIRE].

[31] H.M. Lee, M. Park and V. Sanz, Interplay between Fermi gamma-ray lines and collider searches, JHEP 03 (2013) 052 [arXiv:1212.5647] [INSPIRE].

[32] LAT collaboration, W.B. Atwood et al., The Large Area Telescope on the Fermi Gamma-ray Space Telescope Mission, Astrophys. J. 697 (2009) 1071 [arXiv:0902.1089] [INSPIRE].

[33] A.A. Abdo et al., Fermi LAT Search for Photon Lines from 30 to $200 \mathrm{GeV}$ and Dark Matter Implications, Phys. Rev. Lett. 104 (2010) 091302 [arXiv:1001.4836] [INSPIRE].

[34] LAT collaboration, M. Ackermann et al., Fermi LAT Search for Dark Matter in Gamma-ray Lines and the Inclusive Photon Spectrum, Phys. Rev. D 86 (2012) 022002 [arXiv: 1205.2739] [INSPIRE].

[35] H.E.S.S. collaboration, A. Abramowski et al., Search for photon line-like signatures from Dark Matter annihilations with H.E.S.S, Phys. Rev. Lett. 110 (2013) 041301 [arXiv: 1301.1173] [INSPIRE].

[36] Fermi-LAT collaboration, M. Ackermann et al., Constraining Dark Matter Models from a Combined Analysis of Milky Way Satellites with the Fermi Large Area Telescope, Phys. Rev. Lett. 107 (2011) 241302 [arXiv:1108.3546] [INSPIRE].

[37] Fermi-LAT collaboration, M. Ackermann et al., Dark Matter Constraints from Observations of 25 Milky Way Satellite Galaxies with the Fermi Large Area Telescope, Phys. Rev. D 89 (2014) 042001 [arXiv:1310.0828] [InSPIRE].

[38] W. Buchmüller and M. Garny, Decaying vs Annihilating Dark Matter in Light of a Tentative Gamma-Ray Line, JCAP 08 (2012) 035 [arXiv: 1206.7056] [INSPIRE].

[39] T. Cohen, M. Lisanti, T.R. Slatyer and J.G. Wacker, Illuminating the $130 \mathrm{GeV}$ Gamma Line with Continuum Photons, JHEP 10 (2012) 134 [arXiv:1207.0800] [INSPIRE].

[40] I. Cholis, M. Tavakoli and P. Ullio, Searching for the continuum spectrum photons correlated to the $130 \mathrm{GeV}$ gamma-ray line, Phys. Rev. D 86 (2012) 083525 [arXiv: 1207.1468] [INSPIRE]. 
[41] X. Huang, Q. Yuan, P.-F. Yin, X.-J. Bi and X. Chen, Constraints on the dark matter annihilation scenario of Fermi $130 \mathrm{GeV} \gamma$-ray line emission by continuous gamma-rays, Milky Way halo, galaxy clusters and dwarf galaxies observations, JCAP 11 (2012) 048 [Erratum ibid. 1305 (2013) E02] [arXiv:1208.0267] [INSPIRE].

[42] M. Asano, T. Bringmann, G. Sigl and M. Vollmann, The 130 GeV gamma-ray line and generic dark matter model building constraints from continuum gamma rays, radio and antiproton data, Phys. Rev. D 87 (2013) 103509 [arXiv: 1211.6739] [INSPIRE].

[43] D. Hooper, C. Kelso and F.S. Queiroz, Stringent and Robust Constraints on the Dark Matter Annihilation Cross section From the Region of the Galactic Center, Astropart. Phys. 46 (2013) 55 [arXiv:1209.3015] [INSPIRE].

[44] PAMELA collaboration, O. Adriani et al., PAMELA results on the cosmic-ray antiproton flux from $60 \mathrm{MeV}$ to $180 \mathrm{GeV}$ in kinetic energy, Phys. Rev. Lett. 105 (2010) 121101 [arXiv: 1007.0821 ] [INSPIRE].

[45] G. Bélanger, C. Boehm, M. Cirelli, J. Da Silva and A. Pukhov, PAMELA and FERMI-LAT limits on the neutralino-chargino mass degeneracy, JCAP 11 (2012) 028 [arXiv:1208.5009] [INSPIRE].

[46] X. Chu, T. Hambye, T. Scarna and M.H.G. Tytgat, What if Dark Matter Gamma-Ray Lines come with Gluon Lines?, Phys. Rev. D 86 (2012) 083521 [arXiv:1206.2279] [INSPIRE].

[47] H.M. Lee, M. Park and V. Sanz, Gravity-mediated (or Composite) Dark Matter, Eur. Phys. J. C 74 (2014) 2715 [arXiv: 1306.4107] [InSPIRE].

[48] L. Randall and R. Sundrum, A large mass hierarchy from a small extra dimension, Phys. Rev. Lett. 83 (1999) 3370 [hep-ph/9905221] [INSPIRE].

[49] L. Randall, V. Sanz and M.D. Schwartz, Entropy area relations in field theory, JHEP 06 (2002) 008 [hep-th/0204038] [INSPIRE].

[50] C. Csáki, Y. Shirman and J. Terning, A Seiberg Dual for the MSSM: Partially Composite $W$ and Z, Phys. Rev. D 84 (2011) 095011 [arXiv:1106.3074] [inSPIRE].

[51] J. Erlich, E. Katz, D.T. Son and M.A. Stephanov, QCD and a holographic model of hadrons, Phys. Rev. Lett. 95 (2005) 261602 [hep-ph/0501128] [INSPIRE].

[52] L. Da Rold and A. Pomarol, Chiral symmetry breaking from five dimensional spaces, Nucl. Phys. B 721 (2005) 79 [hep-ph/0501218] [INSPIRE].

[53] J. Hirn and V. Sanz, Interpolating between low and high energy QCD via a $5 D$ Yang-Mills model, JHEP 12 (2005) 030 [hep-ph/0507049] [INSPIRE].

[54] J. Hirn, N. Rius and V. Sanz, Geometric approach to condensates in holographic QCD, Phys. Rev. D 73 (2006) 085005 [hep-ph/0512240] [INSPIRE].

[55] C. Csáki, J. Hubisz and S.J. Lee, Radion phenomenology in realistic warped space models, Phys. Rev. D 76 (2007) 125015 [arXiv:0705.3844] [INSPIRE].

[56] C. Csáki, M.L. Graesser and G.D. Kribs, Radion dynamics and electroweak physics, Phys. Rev. D 63 (2001) 065002 [hep-th/0008151] [INSPIRE].

[57] J. Fan, W.D. Goldberger, A. Ross and W. Skiba, Standard Model couplings and collider signatures of a light scalar, Phys. Rev. D 79 (2009) 035017 [arXiv:0803.2040] [INSPIRE].

[58] R. Fok, C. Guimaraes, R. Lewis and V. Sanz, It is a Graviton! or maybe not, JHEP 12 (2012) 062 [arXiv: 1203.2917] [INSPIRE]. 
[59] A.L. Fitzpatrick, J. Kaplan, L. Randall and L.-T. Wang, Searching for the Kaluza-Klein Graviton in Bulk RS Models, JHEP 09 (2007) 013 [hep-ph/0701150] [INSPIRE].

[60] T. Gherghetta and A. Pomarol, Bulk fields and supersymmetry in a slice of AdS, Nucl. Phys. B 586 (2000) 141 [hep-ph/0003129] [INSPIRE].

[61] T. Gherghetta and A. Pomarol, A warped supersymmetric standard model, Nucl. Phys. B 602 (2001) 3 [hep-ph/0012378] [INSPIRE].

[62] Y. Grossman and M. Neubert, Neutrino masses and mixings in nonfactorizable geometry, Phys. Lett. B 474 (2000) 361 [hep-ph/9912408] [INSPIRE].

[63] H. Davoudiasl, J.L. Hewett and T.G. Rizzo, Bulk gauge fields in the Randall-Sundrum model, Phys. Lett. B 473 (2000) 43 [hep-ph/9911262] [INSPIRE].

[64] A. Pomarol, Gauge bosons in a five-dimensional theory with localized gravity, Phys. Lett. B 486 (2000) 153 [hep-ph/9911294] [INSPIRE].

[65] S. Chang, J. Hisano, H. Nakano, N. Okada and M. Yamaguchi, Bulk standard model in the Randall-Sundrum background, Phys. Rev. D 62 (2000) 084025 [hep-ph/9912498] [InSPIRE].

[66] G.F. Giudice, R. Rattazzi and J.D. Wells, Graviscalars from higher dimensional metrics and curvature Higgs mixing, Nucl. Phys. B 595 (2001) 250 [hep-ph/0002178] [INSPIRE].

[67] H. Davoudiasl, J.L. Hewett and T.G. Rizzo, Experimental probes of localized gravity: On and off the wall, Phys. Rev. D 63 (2001) 075004 [hep-ph/0006041] [INSPIRE].

[68] K. Agashe, H. Davoudiasl, G. Perez and A. Soni, Warped Gravitons at the LHC and Beyond, Phys. Rev. D 76 (2007) 036006 [hep-ph/0701186] [INSPIRE].

[69] R. Bao, M.S. Carena, J. Lykken, M. Park and J. Santiago, Revamped braneworld gravity, Phys. Rev. D 73 (2006) 064026 [hep-th/0511266] [inSPIRE].

[70] M.S. Carena, T.M.P. Tait and C.E.M. Wagner, Branes and orbifolds are opaque, Acta Phys. Polon. B 33 (2002) 2355 [hep-ph/0207056] [INSPIRE].

[71] Y. Cui, T. Gherghetta and J.D. Wells, Emergent Electroweak Symmetry Breaking with Composite W, Z Bosons, JHEP 11 (2009) 080 [arXiv:0907.0906] [INSPIRE].

[72] A. Ibarra, S. Lopez Gehler and M. Pato, Dark matter constraints from box-shaped gamma-ray features, JCAP 07 (2012) 043 [arXiv:1205. 0007] [INSPIRE].

[73] J. Fan and M. Reece, Simple dark matter recipe for the 111 and 128 GeV Fermi-LAT lines, Phys. Rev. D 88 (2013) 035014 [arXiv:1209.1097] [InSPIRE].

[74] A. Ibarra, H.M. Lee, S. López Gehler, W.-I. Park and M. Pato, Gamma-ray boxes from axion-mediated dark matter, JCAP 05 (2013) 016 [arXiv:1303.6632] [INSPIRE].

[75] S. Baek and H. Okada, Hidden sector dark matter with global $\mathrm{U}(1)_{X}$-symmetry and Fermi-LAT $130 \mathrm{GeV} \gamma$-ray excess, Phys. Lett. B 728 (2014) 630 [arXiv:1311.2380] [INSPIRE].

[76] C. Garcia-Cely, A. Ibarra and E. Molinaro, Cosmological and astrophysical signatures of dark matter annihilations into pseudo-Goldstone bosons, JCAP 02 (2014) 032 [arXiv: 1312.3578] [INSPIRE].

[77] J. Hirn and V. Sanz, (Not) Summing over Kaluza-Kleins, Phys. Rev. D 76 (2007) 044022 [hep-ph/0702005] [INSPIRE]. 
[78] C. Csáki, M. Graesser, L. Randall and J. Terning, Cosmology of brane models with radion stabilization, Phys. Rev. D 62 (2000) 045015 [hep-ph/9911406] [INSPIRE].

[79] Planck collaboration, P.A.R. Ade et al., Planck 2013 results. XVI. Cosmological parameters, arXiv:1303.5076 [INSPIRE].

[80] Y. Mambrini, Higgs searches and singlet scalar dark matter: Combined constraints from XENON 100 and the LHC, Phys. Rev. D 84 (2011) 115017 [arXiv:1108.0671] [InSPIRE].

[81] ATLAS collaboration, Search for invisible decays of a Higgs boson produced in association with a Z boson in ATLAS, ATLAS-CONF-2013-011 (2013).

[82] CMS collaboration, Search for invisible Higgs produced in association with a $Z$ boson, CMS-PAS-HIG-13-018.

[83] XENON100 collaboration, E. Aprile et al., Dark Matter Results from 225 Live Days of XENON100 Data, Phys. Rev. Lett. 109 (2012) 181301 [arXiv:1207.5988] [INSPIRE].

[84] LUX collaboration, D.S. Akerib et al., First results from the LUX dark matter experiment at the Sanford Underground Research Facility, Phys. Rev. Lett. 112 (2014) 091303 [arXiv: 1310.8214] [INSPIRE].

[85] J. Kopp, E.T. Neil, R. Primulando and J. Zupan, From gamma ray line signals of dark matter to the LHC, Phys. Dark Univ. 2 (2013) 22 [Erratum ibid. 2 (2013) 176-177] [arXiv:1301.1683] [INSPIRE].

[86] J.M. Cline, G. Dupuis and Z. Liu, LHC constraints on dark matter with (130 GeV) gamma ray lines, JHEP 09 (2013) 065 [arXiv:1306.3217] [INSPIRE].

[87] ATLAS collaboration, Search for dark matter candidates and large extra dimensions in events with a photon and missing transverse momentum in pp collision data at $\sqrt{s}=7 \mathrm{TeV}$ with the ATLAS detector, Phys. Rev. Lett. 110 (2013) 011802 [arXiv:1209.4625] [INSPIRE].

[88] CMS collaboration, Search for Dark Matter and Large Extra Dimensions in pp Collisions Yielding a Photon and Missing Transverse Energy, Phys. Rev. Lett. 108 (2012) 261803 [arXiv: 1204.0821] [INSPIRE].

[89] J. Ellis, V. Sanz and T. You, Associated Production Evidence against Higgs Impostors and Anomalous Couplings, Eur. Phys. J. C 73 (2013) 2507 [arXiv:1303.0208] [InSPIRE].

[90] J. Ellis, R. Fok, D.S. Hwang, V. Sanz and T. You, Distinguishing 'Higgs' spin hypotheses using $\gamma \gamma$ and $W W^{*}$ decays, Eur. Phys. J. C 73 (2013) 2488 [arXiv:1210.5229] [INSPIRE].

[91] J. Ellis, D.S. Hwang, V. Sanz and T. You, A fast track towards the 'Higgs' spin and parity, JHEP 11 (2012) 134 [arXiv:1208.6002] [InSPIRE].

[92] J. Jaeckel, M. Jankowiak and M. Spannowsky, LHC probes the hidden sector, Phys. Dark Univ. 2 (2013) 111 [arXiv: 1212.3620] [INSPIRE].

[93] M. Gouzevitch, A. Oliveira, J. Rojo, R. Rosenfeld, G.P. Salam and V. Sanz, Scale-invariant resonance tagging in multijet events and new physics in Higgs pair production, JHEP $\mathbf{0 7}$ (2013) 148 [arXiv: 1303.6636] [INSPIRE].

[94] G. Bélanger, M. Heikinheimo and V. Sanz, Model-Independent Bounds on Squarks from Monophoton Searches, JHEP 08 (2012) 151 [arXiv: 1205.1463] [INSPIRE]. 